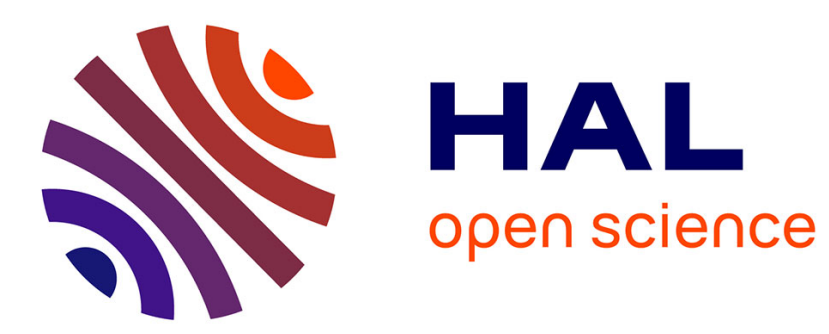

\title{
Characterization and upscaling of hydrodynamic transport in heterogeneous dual porosity media
}

Philippe Gouze, Alexandre Puyguiraud, Delphine Roubinet, Marco Dentz

\section{To cite this version:}

Philippe Gouze, Alexandre Puyguiraud, Delphine Roubinet, Marco Dentz. Characterization and upscaling of hydrodynamic transport in heterogeneous dual porosity media. Advances in Water Resources, 2020, 146, 10.1016/j.advwatres.2020.103781 . hal-02999509

\section{HAL Id: hal-02999509 \\ https://hal.science/hal-02999509}

Submitted on 10 Nov 2020

HAL is a multi-disciplinary open access archive for the deposit and dissemination of scientific research documents, whether they are published or not. The documents may come from teaching and research institutions in France or abroad, or from public or private research centers.
L'archive ouverte pluridisciplinaire HAL, est destinée au dépôt et à la diffusion de documents scientifiques de niveau recherche, publiés ou non, émanant des établissements d'enseignement et de recherche français ou étrangers, des laboratoires publics ou privés. 


\title{
Characterization and upscaling of hydrodynamic transport in heterogeneous dual porosity media
}

\author{
Philippe Gouze ${ }^{\mathrm{a}, *}$, Alexandre Puyguiraud ${ }^{\mathrm{b}}$, Delphine Roubinet ${ }^{\mathrm{a}}$, Marco Dentz ${ }^{\mathrm{b}}$ \\ a Géosciences, Université de Montpellier, CNRS, Montpellier, France \\ ${ }^{\mathrm{b}}$ Spanish National Research Council (IDAEA-CSIC), 08034 Barcelona, Spain
}

\section{A R T I C L E I N F O}

\section{Keywords:}

Non-Fickian dispersion

Heterogeneous porous media

Upscaling

Time domain random walk

Continuous time random walk

Dual multirate mass transfer model

\begin{abstract}
A B S T R A C T
We study the upscaling of pore-scale transport of passive solute in a carbonate rock sample. It is characterized by microporous regions displaying heterogeneous porosity distribution that are accessible due to diffusion only, and a strongly heterogeneous mobile pore space, characterized by a broad distribution of flow velocities. We observe breakthrough curves that are characterized by strong tailing, which can be attributed to velocity variability in the flowing medium portion, and solute retention in the microporous space. Using accurate numerical flow and transport simulations, we separate these two mechanisms by analyzing the statistics of residence times in the mobile phase, and the trapping and residence time statistics in the mmobile phase. We employ a continuous time random walk framework in order to upscale transport using a particle based implementation of mobile-immobile mass transfer, and heterogeneous advection. This approach is based on the statistics of the characteristic mobile and immobile residence times, and mass transfer rates between the two continua. While classical mobile-immobile approaches model mass transfer as a constant rate process, we find that the trapping rate increases with increasing mobile residence times until it reaches a constant asymptotic value. Based on these findings and the statistical characteristics of travel and retention times, we derive an upscaled Lagrangian transport model that separates the processes of heterogeneous advection and diffusion in the immobile microporous space, and provides accurate descriptions of the observed non-Fickian breakthrough curves. These results shed light on transport upscaling in highly complex dual-porosity rocks for which mobile-immobile mass transfer are controlled by a dual multirate process controlled by the heterogeneity of both the flow field in the connected porosity and the diffusion in the no-flow regions.
\end{abstract}

\section{Introduction}

Solute transport in the laminar flow through the void space of a porous medium is due to molecular diffusion and advection. Despite the simplicity of these fundamental processes, observed transport is characterized by complex features such as strong breakthrough curve tailing, non-Gaussian concentration distributions, anomalous dispersion, incomplete mixing, and intermittent Lagrangian flow properties (Cortis and Berkowitz, 2004; Seymour et al., 2004; Bijeljic et al., 2011; De Anna et al., 2013; Bijeljic et al., 2013; Kang et al., 2014; Holzner et al., 2015; Morales et al., 2017). These behaviors are due to the intricate structure of the pore space, and the multi-scale heterogeneity distribution, which cause broad distributions of advective and diffusive mass transfer time scales and transport pathways (Bijeljic et al., 2011; Porta et al., 2015; Puyguiraud et al., 2019a). The understanding of these heterogeneity mechanisms, and their quantification in upscaled transport models are key issues in many academic and engineering applications concerned

\footnotetext{
* Corresponding author.

E-mail address: Philippe.Gouze@UMontpellier.fr (P. Gouze).
}

with the large scale (macroscopic) prediction of the fate of conservative and reactive solutes in geological and engineered media, such as the assessment of groundwater contamination and remediation, geological storage of nuclear waste, geothermal energy production, and underground storage of carbon dioxide (Domenico and Schwartz, 1997; Poinssot and Geckeis, 2012; Niemi et al., 2017).

Classical upscaling approaches quantify Darcy scale transport in terms of hydrodynamic dispersion coefficients (Bear, 1972), which incorporate the large scale dispersive effect of pore-scale velocity fluctuations. The key issue is evidently the determination of the macroscopic dispersion coefficient. For instance, de Josselin de Jong (1958) and Saffman (1959) used Lagrangian stochastic models for pore-scale particle motion in order to derive expressions for the hydrodynamic dispersion coefficients. Their approaches are based on the fact that velocities vary on typical length scales, the pore lengths. Thus, particles spend more time in low than in high flow velocity regions. This behavior, which lies at the origin of pore-scale Lagrangian intermittency (Dentz et al., 2016), is modeled by a distribution of travel 
times. The spatial transitions and the transition times depend on both the pore velocities and molecular diffusion. Since these pioneering works, hydrodynamic dispersion and its dependence on the local Péclet number (the ratio of the characteristic diffusion time to the advection time) was the subject of numerous experimental, numerical and theoretical investigations (Scheven, 2013; Swanson et al., 2015; Pfannkuch, 1963; Rashidi et al., 1996; Jourak et al., 2013; Bijeljic and Blunt, 2006). Systematic upscaling approaches have been based on generalized Taylor dispersion theory (Brenner, 1980; Salles et al., 1993), volume averaging (Quintard and Whitaker, 1994; Davit et al., 2012; 2013) and continuous time random walk (CTRW) (Bijeljic and Blunt, 2006).

CTRW methods similar to the approaches involved in the works of de Josselin de Jong (1958) and Saffman (1959) were used to model non-Fickian pore-scale transport features such as anomalous dispersion, breakthrough curves tailing and intermittent Lagrangian particle velocities (Bijeljic et al., 2011; De Anna et al., 2013; Gjetvaj et al., 2015; Kang et al., 2014; Puyguiraud et al., 2019b). The implementation of CTRW is often handled by modeling particle transport through transitions over fixed spatial scales characterized by random transition times (Berkowitz et al., 2006). The time domain random walk (TDRW) method that will be used in this study, is also based on particles motion over fixed distance. Transition times are determined kinematically from the Eulerian flow field and the spatially distributed properties, for instance the porosity and diffusivity, that can be mapped either from tomographic imaging or from (statistical) models. Thus, these methods provide a means to relate pore-scale flow properties to Darcy scale transport behavior (Bijeljic et al., 2011; Puyguiraud et al., 2019, 2020).

The presence of immobile medium regions that consist of dead end pores, regions of low flow in the wake of solid grains and microporosity where diffusion dominates are common features of porous media such as reservoir rocks. The large difference in terms of Péclet number between these zones of the porous media and the connected network of pores that forms the usual macroporosity (the flowing porosity) supports the use of dual continuum mobile-immobile mass transfer approaches first proposed by van Genuchten and Wierenga (1976). This approach has been widely used to take into account the often-encountered control of spatially distributed diffusive zones on the overall hydrodynamic transport and specifically on the occurrence of often highly non-Fickian breakthrough curves observed experimentally from laboratory to field scales. The heterogeneous medium is modeled by overlapping mobile and immobile continua. At each point in space, the system state is defined by a mobile and series of immobile concentrations. The mobile and immobile continua communicate through linear mass transfer in twoequation models (Ahmadi et al., 1998; Cherblanc et al., 2007), which can be formulated in a way that allows the immobile concentrations to be written as linear functionals of the mobile concentration, which are characterized by a memory kernel (Haggerty and Gorelick, 1995; Carrera et al., 1998) that accounts for the microscale mass transfer processes. As such, it is a method to upscale pore-scale transport. Many implementations of this approach consider a constant average velocity in the mobile medium portion (Liu and Kitanidis, 2012; Porta et al., 2013; 2015). However, advective heterogeneity, this means velocity variability in the flowing medium portion, by itself gives rise to anomalous transport (Bijeljic et al., 2011; De Anna et al., 2013; Kang et al., 2014; Puyguiraud et al., 2019). This is why the importance of pore-scale velocity statistics and their relation to the complex medium and heterogeneity structure have been studied in a series of recent experimental and numerical works (Siena et al., 2014; Matyka et al., 2016; Holzner et al., 2015; De Anna et al., 2017; Alim et al., 2017; Dentz et al., 2018; Aramideh et al., 2018).

Some authors have coupled CTRW models of advective heterogeneity with trapping in immobile regions (Gjetvaj et al., 2015; Dentz et al., 2018). Key items for these modeling approaches are the identification of the dominant pore-scale transport and mass transfer processes, their relation to the pore-scale medium and the flow properties. These aims have been pursued by experiments (Swanson et al., 2015), numeri- cal simulations (de Vries et al., 2017; Ceriotti et al., 2019) and formal upscaling using volume averaging (Davit et al., 2012; Orgogozo et al., 2013; Porta et al., 2015), and Lagrangian CTRW based methods (Gjetvaj et al., 2015; Dentz et al., 2018). Volume averaging delineates a mobile region, the flowing porosity, and immobile regions such as biofilms (Orgogozo et al., 2013) based on a velocity cutoff determined from a Péclet criterion (Porta et al., 2015). The large scale transport model is then obtained by averaging the microscale transport equations over a unit cell that is statistically representative of the properties of the medium and the flow, and contains two distinct medium portions, which, as outlined above, are connected through mass transfer across domain boundaries. Lagrangian stochastic models (Margolin et al., 2003; Benson and Meerschaert, 2009; Dentz et al., 2012; Comolli et al., 2016) formulate mass transfer between mobile and immobile medium regions through compound stochastic Poisson processes (Feller, 1968). This means that mass transfer events occur at constant rate, quantified by the Poisson process, which renders the residence time in immobile regions as the sum over individual trapping times a compound Poisson process. As shown by Margolin et al. (2003), Benson and Meerschaert (2009) and discussed further in this paper, this formulation is equivalent to Eulerian mobile-immobile mass transfer formulations.

This study aims at testing our capability of characterizing and upscaling hydrodynamic transport in heterogeneous natural reservoirs where both velocity distribution and immobile domain heterogeneity cause anomalous transport, starting from the model assuming that mobileimmobile mass transfers are controlled by a Poisson process. For that we use as an example of highly heterogeneous media, a carbonate sample imaged using X-Ray microtomography that displays marked bimodal structural heterogeneity caused by the presence of connected macroporosity and microporous material that results from grain sedimentation and diagenesis events. The image is processed in order to map the mobile and the immobile domain and direct numerical simulations of flow and transport are performed. We investigate in detail the statistics of mobile and immobile particle motion in terms of the respective residence times, the trapping rates and the mobile and immobile times between trapping events. Using the detailed statistical analysis, we discuss the salient features of transport at the pore scale, and quantify them in an upscaled transport model based on a Lagrangian formulation that implements in a simple form the specific process that characterizes the spatial distribution of the mobile-immobile mass transfers in heterogeneous media.

The paper is organized as follows. Section 2 details the methodology. It describes the numerical solution of the direct flow and transport problem, the simulation setup, the boundary conditions and the model outputs which allow investigating in detail the statistics of mobile and immobile particle motion in terms of the respective residence times, the trapping rates and the mobile and immobile times between trapping events. In Section 3 we consider transport in a simple fracture matrix setup in order to present the concept of the basic Lagrangian methodology for a simple mobile-immobile system for which the single trapping rate upscaling CTRW formulation is detailed and then validated using the direct simulation results. Then, following the same approaches we investigate in Section 4 the transport behavior in the carbonate rock sample using direct numerical simulations as well as the upscaling of transport in the immobile domain using a statistical multi-trapping approach. Then, in Section 5, we derive a new upscaled Lagrangian model and validate it by comparison with the results of the direct numerical simulations. Conclusions are presented in Section 6.

\section{Material and methods}

\subsection{Sample MC10 properties}

The MC10 carbonate sample is a porous and permeable rock made of quasi pure calcite. The structure that results from complex sedimentation and diagenetic events is made of impervious grains of variable 

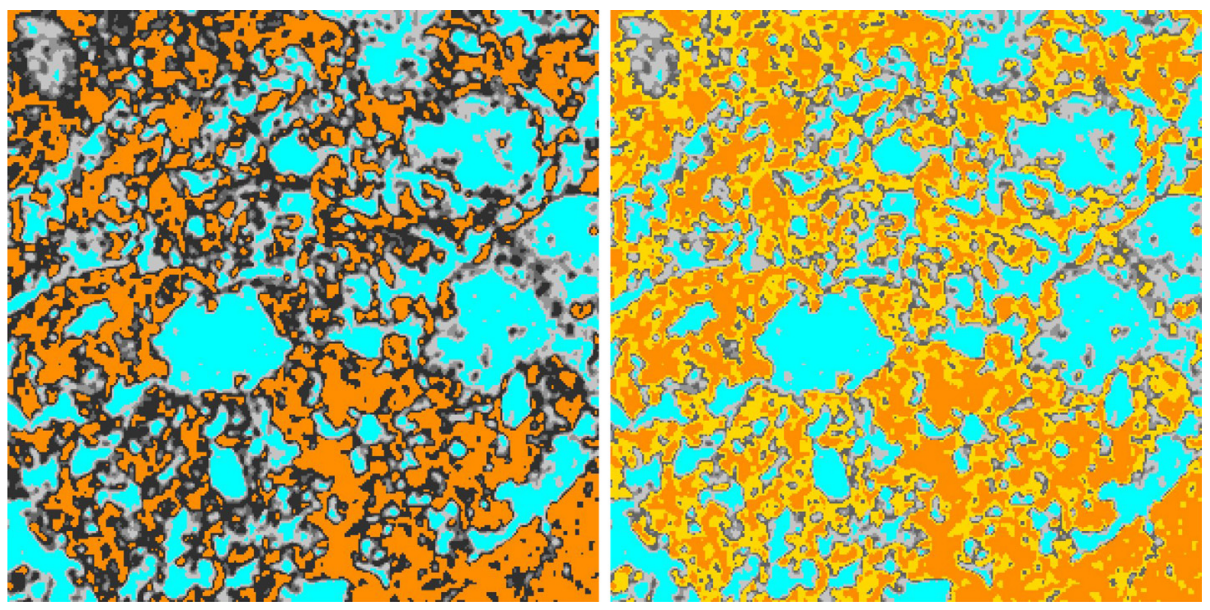

Fig. 1. Porosity map of a slice normal to the main flow direction $z$ positioned at $z=450$. The image on the left displays the porosity map before threshold. Orange and cyan colors denote the solid phase $(\phi=0)$ and the mobile domain $(\phi=1)$ respectively, while gray scale from black (low porosity) to white (high porosity) denotes the immobile domain porosity. The image on the right shows in yellow color the fraction of the immobile domain removed (i.e. transformed into solid) when applying a threshold $\zeta=0.1$ (defined in Section 2.1). (For interpretation of the references to colour in this figure legend, the reader is referred to the web version of this article.)

characteristic length ranging from few tens to about $150 \mu \mathrm{m}$ and microporous material that ensure the cohesion of the rock. When performing X-Ray microtomography of such single solid phase material, the X-Ray energy attenuation integrated over each voxel of the final 3dimensional image denotes the porosity. For this study, we use a cropped sub-volume made of $900 \times 900 \times 900$ cubic voxels of side dimension $d x=1.6867 \times 10^{-6} \mathrm{~m}$. MC10 is characterized by connected macropores of mean size $70 \times 10^{-6} \mathrm{~m}$. The microporous material (diagenetic cement) is considered immobile regarding fluid flow and only accessible to solute by diffusion. It displays variable porosity made by pores that are smaller than the imaging resolution. Accordingly, connected macro-porosity delimits the mobile domain while the microporous material delimits the immobile domain. Details on the sample characteristics and the methodologies applied to process the X-ray tomographic image are given in Appendix A.

The effective diffusion $D_{e}$ in each location of the immobile domain (i.e. in each voxel) is the product of the molecular diffusion $D_{0}$ times the effective porosity $\phi_{e}$,

$D_{e}(\mathbf{x})=D_{0} \phi_{e}(\mathbf{x})=D_{0} \phi(\mathbf{x}) / \kappa(\mathbf{x})$,

where $\kappa$ denotes the immobile domain tortuosity that can be considered as a constant or a function of the immobile domain porosity $\phi$ such as the formulation derived from the electric tortuosity by Archie (1942), $\kappa(\mathbf{x})=\phi(\mathbf{x})^{1-m}$. Accordingly, (1) can be rewritten

$D_{e}(\mathbf{x})=D_{0} \phi(\mathbf{x})^{m}$,

with $m$ ranging from 1 (assuming that there is no tortuosity effect) to about 4.5 in microporous limestones (Gouze et al., 2008). The diffusion coefficient $D_{0}$ is constant for all simulations and set to $10^{-9} \mathrm{~m}^{2} \mathrm{~s}^{-1}$. Note that the porosity of the immobile domain is defined as the porosity accessible by a solute diffusing from the mobile domain, and thus can be different from the total porosity of the immobile domain, for instance if porous zones are embedded in zones considered as non-diffusive as explained below.

For each voxel of the immobile domain transport by diffusion is impossible below a given porosity value. Different approaches using, for instance, percolation theory, critical-path analysis or effective medium approximation theory can be used to evaluate the porosity threshold $\zeta$ below which the system is non-percolating for diffusion (see Hunt and Sahimi, 2017; Hommel et al., 2018 and references herein). For the sample considered here, applying a porosity threshold consists in transforming the fraction of the immobile domain where $\phi<\zeta$ into solid:

$D_{e}(\mathbf{x})= \begin{cases}D_{0} \phi_{e} & \text { for } \phi \geq \zeta \\ 0 & \text { for } \phi<\zeta\end{cases}$

The porosity value of the immobile domain resulting from the image processing ranges from 0.045 to 0.193 with mean porosity 0.108
(Hebert et al., 2015). Fig. 1 displays a cross section (normal to the main flow) in the segmented image of the $900^{3}$-voxel sample where the fraction of immobile domain corresponding to porosity below threshold values of $10 \%$ are enlighten. For instance, applying a porosity threshold of $\zeta=0.1$ acts as removing $39 \%$ of the immobile domain. The mean porosity of the remaining fraction of the immobile domain is then 0.175 . However, applying this threshold does not change noticeably the area of the mobile-immobile interface which is $1.22 \times 10^{5} \mathrm{~m}^{2}$ per $\mathrm{m}^{3}$ of mobile domain when $\zeta=0.0$ and $1.20 \times 10^{5} \mathrm{~m}^{-1}$ when $\zeta=0.1$, i.e. a decrease of $1.64 \%$.

In this paper, different tortuosity models are investigated. Assuming a porosity threshold of $\zeta=0.1$ and tortuosity defined by $\kappa(\phi)=\phi^{1-m}$ with $m=2.5$ is the most realistic model (Garing et al., 2014), but models with $m=1.5$ and 4.5 as well as with a constant tortuosity model $\kappa=1.8$ and $\zeta=0$ are investigated in order to explore the feedback control of the immobile domain diffusivity on the overall solute transport and on the upscaling feasibility. A comprehensive characterization of the diffusion properties according to the assumption made on tortuosity are given in Appendix B.

\subsection{Mobile domain flow}

We consider the flow in the sample at low Reynolds so that the porescale flow velocity $\mathbf{v}(\mathbf{x})$ is solution of the Stokes equation

$\nabla^{2} \mathbf{v}(\mathbf{x})=\frac{1}{\mu} \nabla p(\mathbf{x})$

where $p(\mathbf{x})$ is the fluid pressure. The $900^{3}$ cubic voxels mesh is directly used as the meshed domain for OpenFOAM calculations using a permeameter-like configuration: (i) constant pressure is applied at the inlet $(z=0)$ and the outlet $\left(z=L_{z}\right)$ boundaries where 20-pixels layers of unitary porosity are added in order to obtain an accurate determination of the velocity components at the inlet and outlet of the domain, (ii) the domain is bounded by solid at $x=0, x=L_{x}, y=0$ and $y=L_{y}$, (iii) noslip conditions are applied at the mobile-solid and the immobile-solid domain boundaries. $L_{x}, L_{y}$ and $L_{z}$ denote the domain lengths in the $\mathbf{x}, \mathbf{y}$ and $\mathbf{z}$ directions, respectively.

The flow equations are solved via a finite volume scheme implemented in the SIMPLE algorithm of OpenFOAM (https://cfd.direct/openfoam/user-guide/v7-fvsolution/). This algorithm solves the steady state Stokes equation (4) and continuity equation $\nabla \cdot \mathbf{v}(\mathbf{x})=0$ following an iterative procedure. Convergence is reached when the difference in terms of pressure and velocity components between the current and the previous steps is smaller than a threshold. Once convergence has been reached, we extract the velocity field components that are computed at each of the voxel interface.

The fluid velocity in the direction along the $z$-axis (the main flow direction) displays an asymmetric shape with some negative values 

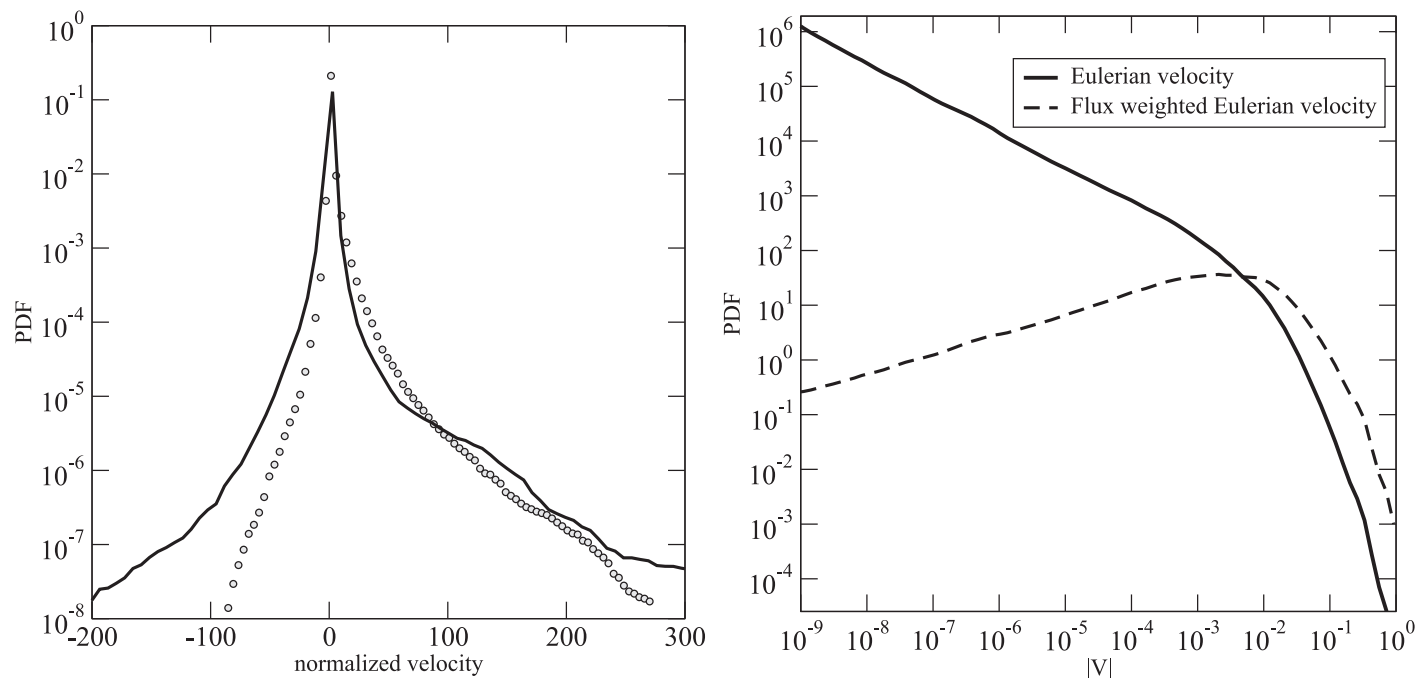

Fig. 2. Left: normalized fluid Eulerian velocity PDF in the direction of the main flow $v_{z} / \overline{v_{z}}$ (circles) and in one of the direction perpendicular to the main flow $v_{x} / \overline{v_{z}}$ (solid line). Right: Eulerian velocity norm $|V|$ PDF (plain line) and flux weighted Eulerian velocity norm PDF (dashed line).

that emphasize the high complexity of the flow field triggered by the high heterogeneity of the mobile domain. The fluid velocity perpendicular to the main flow direction is quasi-symmetric (Fig. 2). The flux weighted velocity norm PDF $P_{E}^{\circ}(|V|)=|V| /\langle|V|\rangle P_{E}(|V|)$, where $P_{E}(|V|)$ denotes the PDF of the Eulerian velocity norm, is displayed in Fig. 2. Puyguiraud et al., 2019 showed that for stationary system $P_{E}^{\circ}$ is equal to the Lagrangian velocity PDF which is the core information required for upscaling advective transport in the mobile domain (Puyguiraud et al., 2019). Upscaling of the advective transport using $P_{E}^{\circ}$ for this highly heterogeneous sample is beyond the scope of the present work that focuses on upscaling the immobile domain transport, and will be presented in a future dedicated paper. Nevertheless, we note that the PDF $P_{E}^{\circ}$ presented in Fig. 2 is quite similar to that of the sandstone sample presented in Fig. 2 in Puyguiraud et al., 2019 for which upscaling methods were proposed by the authors.

\subsection{Transport simulations}

Transport in the mobile-immobile domain is described by the generic advection-diffusion equation which is considered to apply at the scale of each voxel:

$\frac{\partial c(\mathbf{x}, t)}{\partial t}+\mathbf{v}(\mathbf{x}) \cdot \nabla c(\mathbf{x}, t)-D_{e} \nabla^{2} c(\mathbf{x}, t)=0$,

where $D_{e}$ is the effective diffusion coefficient and $\mathbf{v}(\mathbf{x})$ is the flow velocity. In the mobile domain, $D_{e}$ reduces to the molecular diffusion coefficient $D_{0}$ whereas the flow velocity is zero in the immobile domain.

Equation (5) is solved numerically using a time-domain random walk (TDRW) method (Russian et al., 2016), which is based on the formulation of Eq. (5) as a master equation using a finite volume discretization of the spatial operators. A complete description of the TDRW method, a demonstration of its equivalence with Eq. (5), and its implementation using voxelized images of porous media can be found in Dentz et al. (2012) and Russian et al. (2016). The main features of the method are given below. The domain discretization used for transport is the same as the one used for computing the flow. The TDRW approach models the displacement of particles in space and time, their ensemble average giving the solution of the transport equation for the considered media. For each particle, each motion event is denoted by a single jump from one voxel to one of the 6 face-neighboring voxels. As such, the jump distance $\xi$ is constant and equal to the voxel size $d x$. The direction and the jump duration are controlled by the local properties of the voxels, i.e. the fluid velocity and the effective diffusion coefficient. The recursive relations that describe the random walk from position $\mathbf{x}_{j}$ to position $\mathbf{x}_{i}$ of a given particle at jump $n$ is

$\mathbf{x}_{i}(n+1)=\mathbf{x}_{j}(n)+\xi, \quad t(n+1)=t(n)+\tau_{j}$,

with $|\xi|=\xi$ denoting the transition length. The probability $w_{i j}$ for a transition of length $\xi$ from pixel $j$ to pixel $i$, and the transition time $\tau_{j}$ associated to pixel $j$ are given by

$w_{i j}=\frac{b_{i j}}{\sum_{[j k]} b_{k j}}, \quad \tau_{j}=\frac{1}{\sum_{[j k]} b_{k j}}$,

where the notation $\Sigma_{[j k]}$ indicates the summation over the nearest neighbors of pixel $j$. The $b_{i j}$ are given by

$b_{i j}=\frac{\hat{D}_{e i j}}{\xi^{2}}+\frac{\left|v_{i j}\right|}{2 \xi}\left(\frac{v_{i j}}{\left|v_{i j}\right|}+1\right)$,

where $\hat{D}_{e i j}$ denotes the harmonic mean of the diffusion coefficients of pixels $i$ and $j$, and $v_{i j}$ denotes the velocity component of $\mathbf{v}_{j}$ in the direction of pixel $i, v_{i j}=\mathbf{v}_{j} \cdot \xi_{i j}$. As a convention, voxel $i$ is downstream from pixel $j$ if $v_{i j}>0$.

Note that the TDRW can be seen as a continuous time random walk (CTRW) because it treats time as an exponentially distributed continuous random variable whose mean may vary between voxels. In this paper, we use the term TDRW for the numerical random walk method used to solve the direct problem, and the term CTRW for the upscaled random walk framework.

\subsection{TDRW simulations setup}

The applied boundary condition at the sample inlet $(z=0)$ is a pulse of constant concentration in the mobile domain only. This is performed by applying a flux weighted injection of the particles at $t=0$. By construction the pulse is formally an exponential concentration function of characteristic time $\left.\tau_{j}\right|_{z=d x / 2}$ (Russian et al., 2016). The main result is given by the first passage time at the outlet of the mobile domain which denotes the inert tracer breakthrough curve (BTC). No-flux boundary condition is set at $x=0, x=L_{x}, y=0, y=L_{y}$, as well as $z=0$ and $z=L_{z}$ in the immobile domain.

Simulations are performed for different values of Péclet number which is defined by $P e=\langle|V|\rangle l / D_{0}$ where $l$ is a characteristic length which is taken here as the average pore length. Each simulation involves at least $10^{7}$ particles. The statistics concerning the characteristics of the trapping events, such as the trapping time in the immobile domain and the survival time in the mobile domain between two trapping events, are 
obtained by sampling more than $10^{9}$ events (the definition of a trapping event is provided in Section 2.5).

\subsection{Model output}

The mass transfers occurring in the sample are probed by a set of statistical distributions which are given as probability density functions (PDFs), denoted $\check{\psi}$ (.), where the overlying reversed-hat symbol indicates that they are derived from the results of the direct TDRW simulations. These PDFs describe the advection-diffusion transport in the mobile domain, the exchange between the mobile and the immobile domains, and the diffusive transport in the immobile domain. In terms of random walk process, we will name each intrusion of a particle into the immobile domain a "trapping event".

Trapping time PDF denoted $\check{\psi}_{\tau_{i m}}$ is the PDF of the time $\tau_{i m}(p, n(p))$ spent by the particles $p(p=1, ., P)$ in the immobile domain during the trapping events $n(p)(n(p)=1, \ldots, N(p))$, where $P$ is the total number of particles exiting at the sample's outlet and $N(p)$ is the total number of trapping events encountered by particle $p$.

Immobile time PDF denoted $\check{\psi}_{t_{i m}}$ is the PDF of the time $t_{i m}(p)$ spent by the particles in the immobile domain to cross the entire domain (i.e. from $z=0$ to $z=L_{z}$ ). For a given particle $p$, $t_{i m}=\int_{n(p)} \tau_{i m} d n$.

Survival time PDF denoted $\check{\psi}_{\tau_{s}}$ is the PDF of the times $\tau_{s}(p, n(p))$ spent by the particles $p$ in the mobile domain between trapping events $n-1$ and $n$.

Mobile time PDF denoted $\check{\psi}_{t_{m}}$ is the PDF of the time $t_{m}(p)$ spent by the particles in the mobile domain to cross the entire domain. For a given particle $p, t_{m}=\int_{n} \tau_{s} d n+\epsilon$, where $\epsilon$ is the sum of the time spent to move from the inlet to the location of the first trapping event and of the time spent to move from the exit location of trapping event $N(p)$ to the outlet.

Trapping rate PDF denoted $\check{\psi}_{\gamma}$ is the PDF of $\gamma(p)=n(p) / t_{m}(p)$.

First passage time PDF denoted $\check{\psi}_{t_{t}}$ is the PDF of the first passage time $t_{t}(p)$ spent by the particles to cross the domain and is equivalent to the breakthrough curve (BTC). By definition, for each particle $p$

$$
t_{t}(p)=t_{m}(p)+t_{i m}(p)=t_{m}(p)+\sum_{i=1}^{N(p)} \tau_{i m}(p, i) .
$$

3. Modeling transport in a single fracture with mobile-immobile mass transfer

In this section, we investigate the case of the transport of a passive tracer in a simple mobile-immobile domain system that can be adequately represented as a single linear fracture (the mobile domain) crossing a continuous porous matrix (the immobile domain). The different PDFs characterizing the transport process (described in Section 2.5) will be computed using direct TDRW simulations and will later be compared to those resulting from TDRW simulations performed for the MC10 carbonate sample. Furthermore, we present a 1D CTRW model that upscales transport in the fracture matrix system and introduces the main features and concepts used for the upscaling of transport in the MC10 carbonate sample. The detailed fracture-matrix transport model can be formulated in the most general form as

$\phi(y) \frac{\partial c(\mathbf{x}, t)}{\partial t}+u(y) \frac{\partial c(\mathbf{x}, t)}{\partial x}-\nabla \cdot[D(y) \nabla c(\mathbf{x}, t)]=0$,

where $\phi(y)$ is porosity, which is equal to $\phi_{m}$ within the fracture and $\phi_{i m}$ in the matrix; $u(y)$ is the Darcy velocity, which is equal to $u$ in the fracture and 0 in the matrix; similarly, $D(y)$ is the diffusion coefficient, which is equal to $D_{m} \phi_{m}$ in the fracture and equal to $D_{i m} \phi_{i m}$ in the matrix, where $D_{m}$ and $D_{i m}$ denote the diffusion coefficient in the mobile and the immobile domain respectively.

In the following, we consider two equivalent upscaled transport approaches.

\subsection{Upscaling by vertical averaging}

Upscaled transport in this fracture-matrix system can be described by a multirate mass transfer model (Haggerty and Gorelick, 1995; Carrera et al., 1998). In the following, we briefly outline the steps that lead to such a description in order to highlight the underlying assumptions. The upscaled multirate mass transfer description for the fracture-matrix system is obtained by vertical averaging. To this end, we define the averages concentration over the fracture and matrix cross-sections as

$\bar{c}_{m}(x, t)=\frac{1}{d_{m}} \int_{0}^{d_{m}} d y c(\mathbf{x}, t), \quad \bar{c}_{i m}(x, t)=\frac{1}{d_{i m}} \int_{0}^{d_{i m}} d y c(\mathbf{x}, t)$,

where $d_{m}$ is the width of the fracture and $d_{i m}$ of the matrix. Averaging (10) over the fracture cross-section gives

$\phi_{m} \frac{\partial \bar{c}_{m}(x, t)}{\partial t}+u \frac{\partial \bar{c}_{m}(x, t)}{\partial x}-D_{m} \phi_{m} \frac{\partial^{2} \bar{c}_{m}(x, t)}{\partial x^{2}}=-\left.\frac{1}{d_{m}} \phi_{m} D_{m} \frac{\partial c_{m}(\mathbf{x}, t)}{\partial y}\right|_{y=0}$,

while the equation for purely diffusive transport in the matrix domain is

$\phi_{i m} \frac{\partial c_{i m}(\mathbf{x}, t)}{\partial t}-D_{i m} \phi_{i m} \frac{\partial^{2} c_{i m}(\mathbf{x}, t)}{\partial y^{2}}=0$.

The boundary condition are $c_{i m}(x, y=0, t)=c_{m}(x, y=0, t)$ as an expression of concentration continuity. We approximate $c_{m}(\mathbf{x}, t) \approx \bar{c}_{m}(x, t)$, which assumes fast equilibration over the fracture cross-section. Using flux continuity across the fracture matrix interface, we obtain in Appendix C

$\frac{\partial \bar{c}_{m}(x, t)}{\partial t}+v \frac{\partial \bar{c}_{m}(x, t)}{\partial x}-D_{m} \frac{\partial^{2} \bar{c}_{m}(x, t)}{\partial x^{2}}=-\beta \frac{\partial \bar{c}_{i m}(x, t)}{\partial t}$,

where we defined the capacity coefficient $\beta=d_{i m} \phi_{i m} / d_{m} \phi_{m}$ and the pore velocity $v=u / \phi_{m}$. The average matrix concentration can be expressed as a linear functional of the average fracture concentration (Appendix $\mathrm{C}$ )

$\bar{c}_{i m}(x, t)=\int_{0}^{t} d t^{\prime} \varphi\left(t-t^{\prime}\right) \bar{c}_{m}(x, t)$

The memory function is well known (Carrera et al., 1998), and can be expressed in Laplace space as

$\varphi^{*}(\lambda)=\frac{\tanh \left(\sqrt{\lambda \tau_{D}}\right)}{\sqrt{\lambda \tau_{D}}}$,

where we define the characteristic diffusion time $\tau_{D}=d_{i m}^{2} / D_{i m}$ in the matrix. Combining (14) and (15), we obtain the integro-partialdifferential equation

$\frac{\partial \bar{c}_{m}(x, t)}{\partial t}+v \frac{\partial \bar{c}_{m}(x, t)}{\partial x}-D_{m} \frac{\partial^{2} \bar{c}_{m}(x, t)}{\partial x^{2}}=-\beta \frac{\partial}{\partial t} \int_{0}^{t} d t^{\prime} \varphi\left(t-t^{\prime}\right) \bar{c}_{m}(x, t)$,

which is equivalent to the multirate mass transfer model of Haggerty and Gorelick (1995); Carrera et al. (1998). In the following, we describe the formulation of this upscaled model in a Lagrangian framework.

\subsection{Upscaled lagrangian model}

The upscaled Lagrangian approach models one-dimensional advective-diffusive transport along the fracture, which is interrupted by trapping events that are Poisson distributed. This means that transitions from the fracture to the matrix occur at constant rate $\gamma$, which can be quantified by the diffusion rate over the fracture cross-section. At each trapping event, a particle is trapped for a random time distributed according to $\psi_{i m}(t)$. These are the principal ingredients of the upscaled transport model. In the following, we formulate this model in the TDRW framework.

One-dimensional advective-diffusion particle motion at constant velocity $v$ and diffusion coefficient $D_{m}$ is described by particle transitions 
over the fixed distance $\ell$ by a random time $t_{m}$. The probability $w_{u}$ for upstream particle motion is

$w_{u}=\frac{D_{m} \tau_{v}}{\ell^{2}}$.

The probability for downstream motion is accordingly $w_{d}=1-w_{u}$. The time $\tau_{v}$ is defined by

$\tau_{v}=\frac{\ell / v}{1+2 / P e}, \quad P e=\frac{v \ell}{D_{m}}$.

The transition time $t_{m}$ is exponentially distributed

$\psi_{i m}(t)=\tau_{v} \exp \left(-t / \tau_{v}\right)$

These rules represent mobile transport as a TDRW model for advection-diffusion with constant velocity $v$ and diffusion coefficient $D_{m}$ (Russian et al., 2016). This motion is combined with the trapping rules outlined in the following. During a transition of duration $t_{m}, n_{t}$ trapping events occur, such that the total transition time is given by

$t_{t}=t_{m}+\sum_{i=1}^{n_{t}} \tau_{i m}$

The number $n_{t}$ of trapping events is distributed according to the Poisson distribution

$P(n \mid t)=\frac{(\gamma t)^{n_{t}} \exp (-\gamma t)}{n_{t} !}$,

with mean $\left\langle n_{t}\right\rangle=\gamma t$. Thus, the total transition time $t_{t}$ describes a compound Poisson process. Its PDF $\psi(t)$ can be expressed in Laplace space as (Margolin et al., 2003; Dentz et al., 2012)

$\psi^{*}(\lambda)=\frac{1}{1+\lambda \tau_{v}+\gamma \tau_{v}\left[1-\psi_{i m}^{*}(\lambda)\right]}$.

Laplace transformed quantities are marked by an asterisk, the Laplace variable is denoted by $\lambda$.

In order to show the equivalence of this Lagrangian formulation with the MRMT model (17), we derive in Appendix D for the concentration $c_{m}(x, t)$ in the fracture

$\frac{\partial c_{m}(x, t)}{\partial t}+v \frac{\partial c_{m}(x, t)}{\partial x}-D_{m} \frac{\partial^{2} c_{m}(x, t)}{\partial x^{2}}=-\frac{\partial c_{i m}(x, t)}{\partial t}$.

The concentration $c_{i m}(x, t)$ in the matrix is given by

$c_{i m}(x, t)=\gamma \int_{0}^{t} d t^{\prime} \vartheta\left(t-t^{\prime}\right) c_{m}\left(x, t^{\prime}\right)$,

where the memory kernel $\vartheta(t)$ is defined by

$\vartheta(t)=\int_{t}^{\infty} d t^{\prime} \psi_{i m}\left(t^{\prime}\right)$

It denotes the probability that the trapping time is larger than $t$. Using (25) in (24), we obtain for $c_{m}(x, t)$ the governing equation

$\frac{\partial c_{m}(x, t)}{\partial t}+v \frac{\partial c_{m}(x, t)}{\partial x}-D_{m} \frac{\partial^{2} c_{m}(x, t)}{\partial x^{2}}=-\gamma \frac{\partial}{\partial t} \int_{0}^{t} d t^{\prime} \vartheta\left(t-t^{\prime}\right) c_{m}\left(x, t^{\prime}\right)$.

This equation and equation (17) are equivalent if

$\gamma \vartheta(t) \equiv \beta \varphi(t)$.

We first recall that $\varphi(t)$ is normalized to 1 , which can be seen by taking the limit $\lambda \rightarrow 0$ in (16), while the integral over $\vartheta(t)$ is equal to $\left\langle\tau_{i m}\right\rangle$, the mean trapping time. Thus, we obtain

$\gamma\left\langle\tau_{i m}\right\rangle=\beta$.

This equivalence identifies the trapping rate $\gamma$ and trapping time distribution $\psi_{i m}(t)$ as the key quantities in the upscaled model. Both quantities can be accessed by random walk particle tracking simulations as outlined in Section (2.4). In the following, we use this general framework for the upscaling of transport in the MC10 carbonate sample.

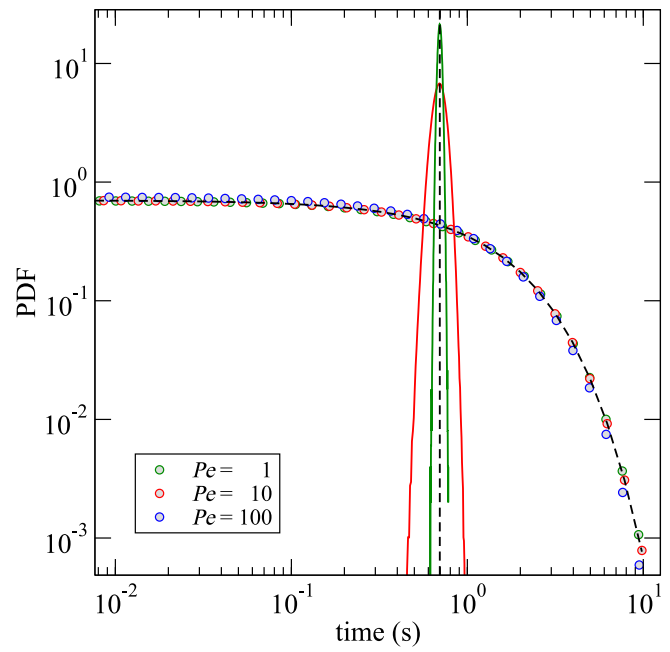

Fig. 3. PDFs of the survival time $\check{\psi}_{\tau}$ computed by TDRW (circles) and the exponential trend (Equation (30)) corresponding to the same mean value $\langle\gamma\rangle=1 /\left\langle\tau_{s}\right\rangle$ $=0.697 \mathrm{~s}^{-1}$ (dashed line) for different Péclet numbers. The colored lines denote the trapping rate PDFs $\check{\psi}_{\gamma}$ where the average $\langle\gamma\rangle=\left\langle n / t_{m}\right\rangle$ (vertical dashed line) is $0.697 \mathrm{~s}^{-1}$.

\subsection{CTRW upscaled model versus TDRW model results}

We tested the CTRW model by comparing the results with direct TDRW simulations for the simplest idealized 2-dimensional representation of a single fracture system. The computational domain is a porous medium (the immobile domain) of dimension $L_{z}=20000$ $\times L_{y}=1001$ pixels embedding a fracture (the mobile domain) of aperture 1 pixel, located at $y=500$, so that the immobile domain depth on each side of the fracture is $\ell_{i m}=500$ pixels. The pixel size is denoted $\xi$ as in Section 2.3.

The flow velocity $v$ in the fracture is constant, the inlet is located at $z=0$ where a pulse injection is applied (see Section 2.4) and the outlet is located at $z=20000$ where the PDF of the first passage time (or breakthrough curve) $\check{\psi}_{t_{t}}$ is monitored. With a fracture aperture $\xi$, the problem is simply characterized by the Péclet number $P e=V \xi / D_{0}$. We performed simulations for constant diffusivity in the immobile domain $\left(D_{e}(x, y)=D\right)$ and for random lognormal distribution with $D_{e}(x, y)$ taken as the spatial geometric mean of the pixel diffusion. Simulations are performed with $\xi=10^{-5} \mathrm{~m}, D_{0}=10^{-9} \mathrm{~m}^{2} \cdot \mathrm{s}^{-1}, D_{e}=1.774 \times 10^{-11} \mathrm{~m}^{2} \cdot \mathrm{s}^{-1}$ and $10^{-12} \leq D_{e}(x, t) \leq 10^{-9} \mathrm{~m}^{2} \cdot \mathrm{s}^{-1}$ for the lognormal distributed diffusion model in the matrix.

A main attribute of the compound Poisson process described in Section 3.2 is that the distribution of the survival time in the mobile domain $\tau_{s}$ is exponentially distributed:

$\psi_{\tau_{s}}(t)=\gamma \exp (-\gamma t)$

where $\gamma=1 /\left\langle\tau_{s}\right\rangle$.

Fig. 3 displays the survival time distribution $\check{\psi}_{\tau_{s}}$ computed from the TDRW which is well fitted by an exponential distribution of mean $\langle\gamma\rangle=$ $\left\langle n / t_{m}\right\rangle=1 /\left\langle\tau_{s}\right\rangle$.

Fig. 4 shows the perfect agreement between the breakthrough curves, or first passage time PDFs $\check{\psi}_{t_{t}}$, resulting from the upscaled CTRW simulations and those obtained from the TDRW simulations, for Pe values ranging from 1 to 100 . The results are similar for the homogeneous immobile domain and for the random lognormal distribution with the same geometric mean diffusion, as expected (Notinger and Estebenet, 2000; Russian et al., 2016). 


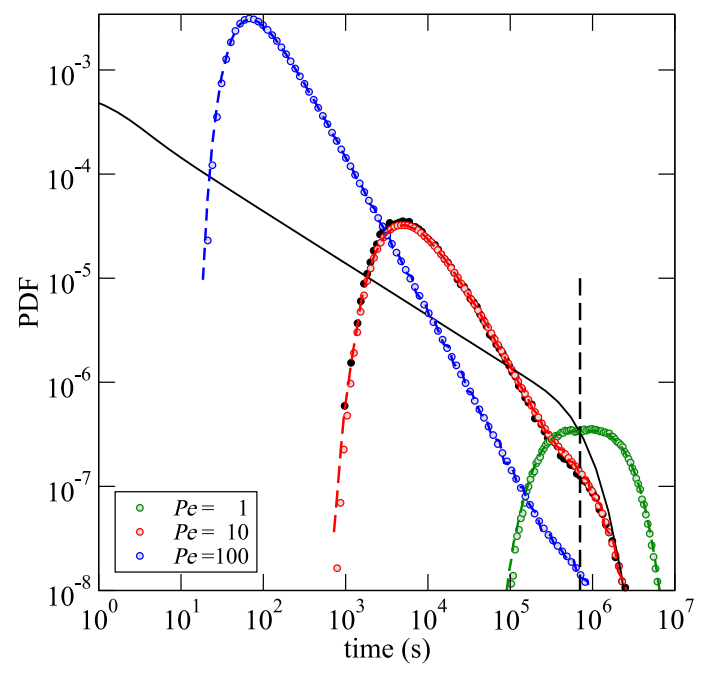

Fig. 4. Green, red and blue circles denote $\check{\psi}_{t}$, the PDFs of the first passage time $t_{t}$ computed by TDRW, for constant $D_{e}=1.774 \times 10^{-11} \mathrm{~m}^{2} \cdot \mathrm{s}^{-1}$ in the immobile domain for $P e=1,10$ and 100, respectively. The black circles, that are almost completely overlapped by the red circles $(P e=10)$, denote $\check{\psi}_{t}$, for random lognormal porosity distribution with geometric mean diffusion equal to $1.774 \times 10^{-11} \mathrm{~m}^{2} \cdot \mathrm{s}^{-1}$. The green, red and blue dashed lines denote the equivalent $\psi_{t_{t}}$ computed by the CTRW. The curve plotted as a continuous black line is the memory function $\varphi(t)$ of slope $-1 / 2$ that characterizes the immobile domain while the vertical black dashed line indicates the diffusion characteristic time $t_{d}=\ell_{i m}^{2} /\left(2 D_{e}\right)=7.044 \times 10^{5} \mathrm{~s}$. (For interpretation of the references to colour in this figure legend, the reader is referred to the web version of this article.)

\section{TDRW modeling of transport in the carbonate sample}

This section concerns direct simulations performed with the TDRW model, i.e. simulations of the 3-dimensional domain. Simulations are performed according to the algorithm and the boundary conditions described in Sections 2.2 and 2.3, respectively. The results presented in this section focus on 4 distinct models that characterize the immobile domain diffusivity distribution in terms of tortuosity $\kappa$ and porosity threshold $\zeta$ (see Table B.1). The simplest model assumes constant tortuosity $\kappa=1.8$ and no porosity threshold, while the three other assume porosity-dependent tortuosity $\kappa=\phi^{m}$, with $m=1.5,2.5$ or 4.5 and a porosity threshold $\zeta=0.1$.

\subsection{Trapping properties of the MC10 sample}

Here, the trapping characteristics of the MC10 sample are analyzed and compared to the CTRW model discussed in Section 3. We recall that this model is characterized by the following feature: the conditional PDF $P_{n}\left(n \mid t_{m}\right)$ that measures the number of trapping events conditioned to the time spent in the mobile domain is a Poisson distribution with a constant trapping rate $\gamma$. This means that the time spent in the mobile domain between two trapping events, or survival time $\tau_{s}$, is characterized by an exponential distribution. The PDF $\check{\psi}_{\tau_{s}}$ computed for the MC10 sample and the one corresponding to Equation (30) with the same average values $\left\langle\tau_{s}\right\rangle$ are displayed in Fig. 5, while the PDF $P_{n}\left(n \mid t_{m}\right)$ computed for the MC10 sample and the one corresponding to Equation (22), where the constant trapping rate $\gamma=\langle\gamma\rangle$, are displayed in Fig. 6 . The latter is obtained by computing the PDF of $n_{t_{m}}$ from Equation (22) for each range of $t_{m}$. The survival time PDF $\check{\psi}_{\tau_{s}}$ does not depend on the average fluid velocity in the sample, i.e. does not depend on the $P e$ value, and is controlled by the transport properties at the mobile-immobile domains interface and thus is controlled by the effective diffusion of the immobile domain in the vicinity of the mobile-immobile interface, that is to say by the local porosity. Fig. 5 shows that the $\check{\psi}_{\tau_{s}}$ PDFs are visually identical when applying a porosity threshold $\zeta \leq 0.1$ or not, emphasiz-

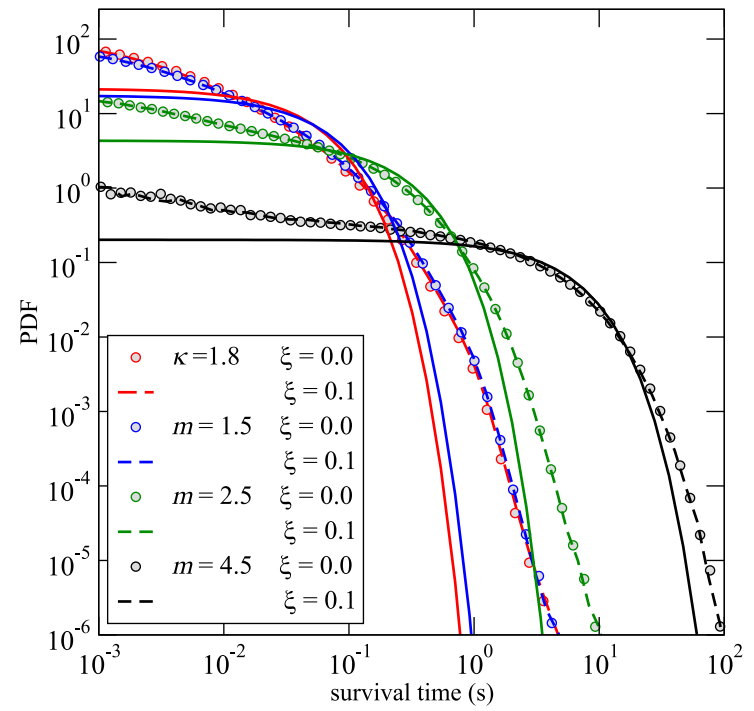

Fig. 5. PDFs of the survival time $\tau_{s}, \check{\psi}_{\tau_{s}}$, for different values of $\kappa$ or alternatively $m$ and $\zeta$. For comparison, the continuous lines denote the exponential trend (Equation (30)) corresponding to the same average values $\left\langle\tau_{s}\right\rangle$.

ing that the value of the porosity threshold does not change noticeably the properties of the immobile domain at the mobile-immobile interface nor its topology, as it is shown also in Appendix B. As expected, $\check{\psi}_{\tau_{s}}$ is strongly shifted toward larger time values when the immobile diffusivity at the mobile-immobile interface decreases. The important point is that $\check{\psi}_{\tau_{s}}$ curves are, as a general rule, not exponential distributions and display an over-representation of the short survival times. We see also larger maximum values compared to what is predicted by the exponential distribution, but this feature decreases when $m$ increases.

The conditional PDF $P_{n}\left(n \mid t_{m}\right)$ resulting from the MC10 simulations is compared to the one computed assuming a Poisson distribution following Eq. 22 with a constant trapping rate $\langle\gamma\rangle$ in Fig. 6 . The conditional PDF $P_{n}\left(n \mid t_{m}\right)$ resulting from the MC10 simulations is noticeably different from the one computed assuming a Poisson distribution with a constant trapping rate. For a given mobile time, the theoretical Poisson model predicts less trapping events than what is measured for the MC10 sample. This discrepancy increases with the value of $t_{m}$.

The trapping rate distribution encompasses the information about the trapping process which is controlled by the complex interactions between the mobile and immobile transport process. As such, one can expect that the trapping rate distribution is a macroscopic observable that characterizes the mobile-immobile mass transfer, and in a similar manner that the memory function is the macroscopic observable that enciphers the entire properties of immobile domain diffusive transport properties. Fig. 7 displays the PDF of $\gamma, \check{\psi}_{\gamma}$ and reports the percentage of the particles that do not encounter trapping when traveling from the inlet to the outlet for different properties of the immobile domain. This percentage depends evidently on the value of the Pe number but also on the properties of the immobile domain. For instance, it ranges from $1.8 \%$ to $95.6 \%$ for $P e=100$ depending on the value of $m$. It follows that the average trapping rate $\langle\gamma\rangle$ cannot be inferred from $1 /\left\langle\tau_{s}\right\rangle$ because the statistics of $\tau_{s}$ concern only particles that encounter trapping whereas a certain number of particles never visit the immobile domain. Yet, interestingly, the results presented in Fig. 7 show that all the PDFs $\breve{\psi}_{\gamma}$ have almost the same average value, $\langle\gamma\rangle=1.51 \pm 0.18 \mathrm{~s}^{-1}$ independently of the immobile domain properties, which means that this value is an intrinsic property of the MC10 sample, probably related to the geometry of the mobile domain and its interface with the immobile domain, despite the distributions $\check{\psi}_{\gamma}$ being strongly dissimilar.

Altogether, these results suggest that the assumptions supporting the CTRW model of Section 3.2 are not strictly met for the carbonate sample 

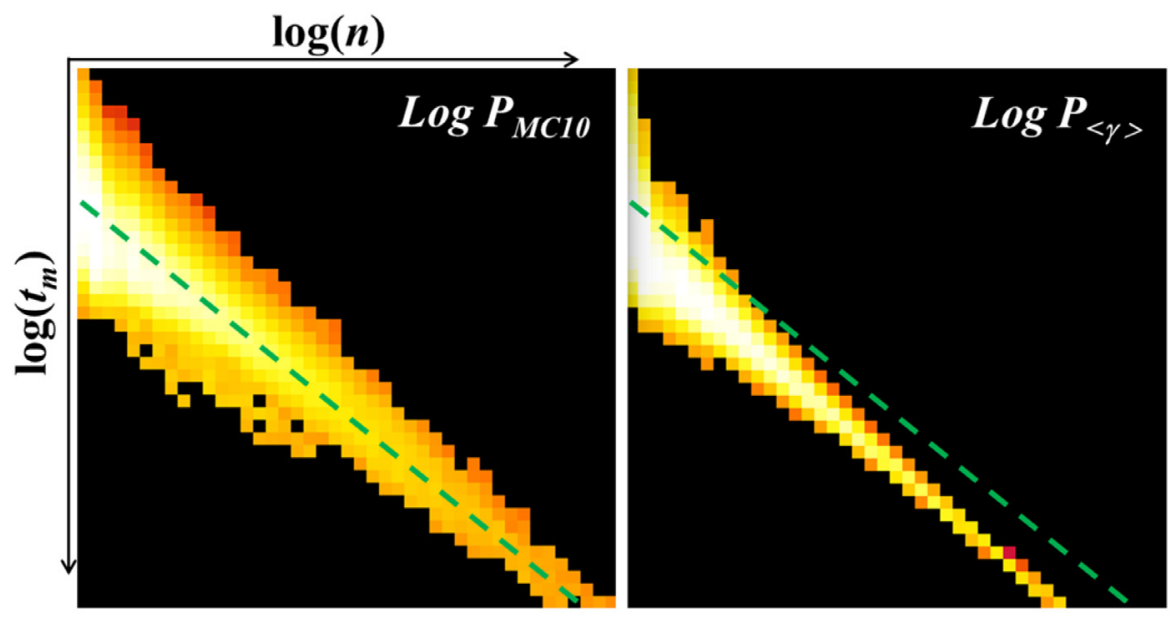

Fig. 6. Left: conditional probability $\log _{10}\left(P_{n}\left(n \mid t_{m}\right)\right)$ obtained for the MC10 sample. Right: conditional probability $\log _{10}\left(P_{n}\left(n \mid t_{m}\right)\right)$ corresponding to the theoretical Poisson distribution computed using a single rate $\langle\gamma\rangle$. Black color indicates values of $\log _{10}\left(P_{n}\left(n \mid t_{m}\right)\right)$ smaller than -5 while color scale from dark red to white denotes values of $\log _{10}\left(P_{n}\left(n \mid t_{m}\right)\right)$ ranging from -5 to -0.68 . The green dashed line is a visual landmark. Results are given for $P e=100, \zeta=0.1$ and $\kappa(\mathbf{x})=\phi(\mathbf{x})^{1-m}$ with $m=2.5$. (For interpretation of the references to colour in this figure legend, the reader is referred to the web version of this article.)

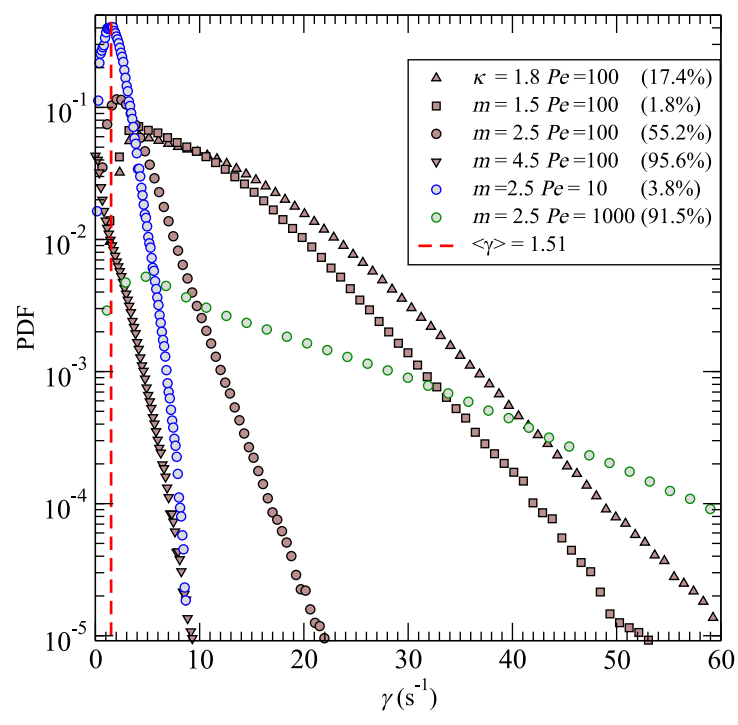

Fig. 7. PDFs of the trapping rate $\check{\psi}_{\gamma}$, where $\gamma(p)=n(p) / t_{m}(p)$ for different properties of the immobile domain and different values of $P e$. The values in parenthesis denote the percentage of particles $p$ traveling the entire domain in the mobile domain without being trapped in the immobile domain. The vertical red discontinuous line indicates the mean trapping rate $\langle\gamma\rangle$ which is equal to $1.51 \pm 0.18$ for all the curves. (For interpretation of the references to colour in this figure legend, the reader is referred to the web version of this article.)

considered here. Taking into account these results, the issue that will be investigated next is to evaluate to which extent the CTRW model is robust enough to model transport in heterogeneous media such as the MC10 sample, or alternatively what additional relationship between the trapping rate properties and the mobile domain properties are required to derive a reliable upscaled model for complex systems such as the MC10 sample.

\subsection{Upscaling the impact of diffusion in the immobile domain}

For each trapping event, the particles that enter the immobile domain at a given location can exit at another location. In the case of the single fracture model with homogeneous equivalent immobile domain, the relocation distance along the linear continuous mobileimmobile interface is a sharp distribution (well described by its mean value 0). Conversely, the relocation of the particles in the MC10 sample is much less predictable due to the strong heterogeneity of the system in which the immobile domain is formed of heterogeneous clus- ters spatially distributed. This is triggered principally by non-continuous mobile-immobile interfaces (lacunar interface) and the possibility of particles to utilize the immobile domain to take a shortcut from a given flow path to another. Conversely, the 1-dimensional CTRW model imposes by construction that particles enter and exit the immobile domain at the same location for each trapping event.

Simulating such a situation while keeping the complete (3dimensional) computation of the transport in the mobile domain is viewed as potentially instructive for understanding conjointly the effect of the particles relocation at the mobile-immobile interface owing to the strong heterogeneity of the interface and the statistical representativeness of the trapping time PDF $\check{\psi}_{\tau_{i m}}$ for modeling the immobile domain transport properties at the scale of the sample. To this end, the TDRW solver is modified such that transport in the mobile domain and the trapping process are kept unchanged, but the time spent in the immobile domain is drawn from the trapping time PDF $\breve{\psi}_{\tau_{i m}}$ previously computed during the corresponding TDRW simulation involving the full direct simulation of the transport in the mobile and the immobile domain. Doing this imposes by construction that particles enter and exit the immobile domain at the same location for each trapping event similarly to the CTRW upscaled model. From now on, the model in which the trapping time PDF is used to model the time spent in the immobile domain at each trapping event is called the UPSCAL TDRW model in contrast to the FULL TDRW model.

\subsubsection{Control of the immobile domain diffusion properties over mobile-immobile mass transfer}

Fig. 8 compiles the main information concerning the results in terms of first passage time $t_{t}$, mobile time $t_{m}$, immobile time $t_{i m}$ and survival time $\tau_{s}$ for the FULL TDRW model and the UPSCAL TDRW model. These data are very valuable for understanding the control of the immobile domain properties on the way particles sample the system. The capacity of the immobile domain to trigger shortcuts between zones of the mobile domain with different flow properties decreases when moving from the $\kappa=1.8-\zeta=0$ model to the $m=2.5-\zeta=0.1$ model and $m=$ $4.5-\zeta=0.1$ model because 1 ) applying a porosity threshold decreases the probability of having immobile domain clusters connected to many pores and 2) increasing the value of $m$ acts as increasing the tortuosity, i.e. the effective diffusion time in the immobile domain (see Table B.1). Consequently, comparing the results for the FULL model with the UP$S C A L$ model for which particles are forced to exit the immobile domain where they entered for each of the trapping events allows not only to understand the feedback effect of the particle relocation process at the mobile-immobile interface on the overall transport that is quantified by the first passage time PDF, but also to decompose the overall transport process in terms of the time spent in the mobile domain and the immobile domain. 

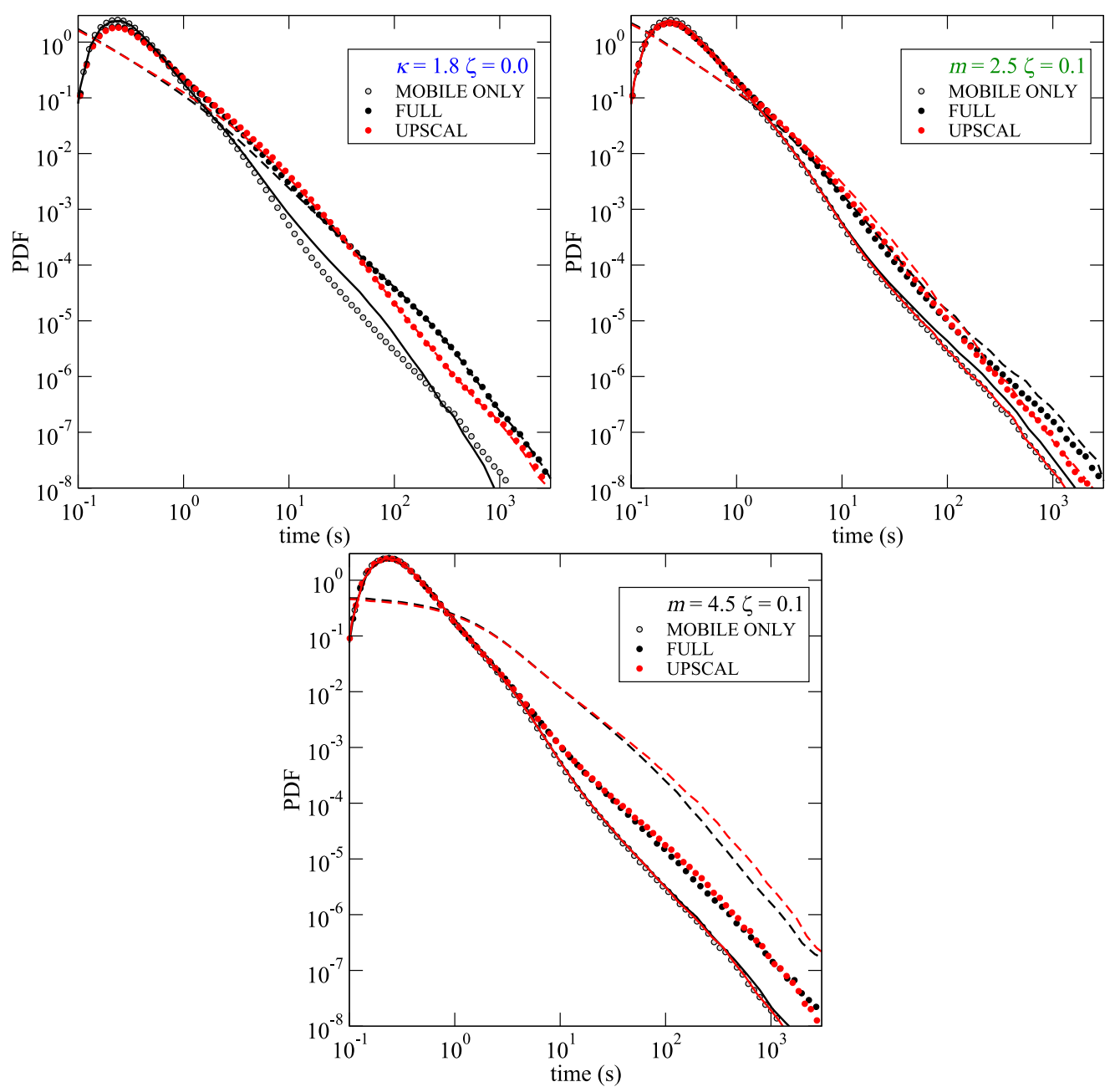

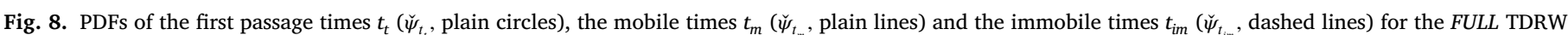

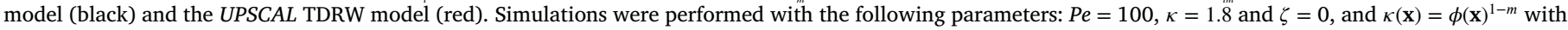

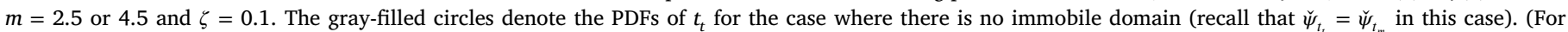
interpretation of the references to colour in this figure legend, the reader is referred to the web version of this article.)

For the $\kappa=1.8-\zeta=0$ model the first passage time PDF $\check{\psi}_{t}$ obtained for the FULL and the UPSCAL model are noticeably dissimilar; their respective shape being fully controlled by the immobile time distribution for intermediate and long times. Conversely, the mobile time PDF are the same but different from the mobile time PDF computed assuming no immobile domain, i.e. depending only on the mobile domain properties. This indicates that the transport is strongly controlled by the broad spatial redistribution of the particles among mobile zones of distinctly different flow rates. As a general rule, one can conclude that the discrepancy between the UPSCAL and the FULL model in terms of first passage time PDF $\check{\psi}_{t_{t}}$ (Fig. 8) originates from the fact that both the trapping rate $\gamma$ and the trapping time in the immobile domain $\tau_{i m}$ are different as a result of the distinct particle relocation processes when encountering trapping events. From these observations, one can speculate that for the $\kappa=1.8-\zeta=0$ model the upscaling of such a system with a one-dimensional model where particles sample the immobile domain according to the ensemble average statistics of the mobile displacement will fail even if one considers a non-unique trapping rate that would be related to the mobile time.

The two other models of immobile domain ( $m=2.5$ and 4.5 ) share the same spatial geometry, i.e. the same boundaries, because they share the same porosity threshold $\zeta=0.1$, but differ from the effective diffusion spatial distribution and mean. Increasing the value of $m$ acts as decreasing 1 ) the mean distance of penetration of the particle into the immobile domain and 2) the relocation distance between the entrance and the exit of the particle in the immobile domain during each trapping event. As such, the model characterized by $m=4.5$ is the most similar to the simple fracture model presented in Section 3.3 in terms of geometry. Indeed, the results presented in Fig. 8 for $m=4.5-\zeta=0.1$ show that the first passage time PDFs $\check{\psi}_{t_{t}}$ are almost similar for the FULL and the UPSCAL models, while the mobile time PDFs of $t_{m}$ ( $\check{\psi}_{t_{m}}$ ) overlap the PDFs of $t_{m}$ for the case where there is no immobile domain. This means that the immobile domain heterogeneity does not control the advective transport in the mobile domain similarly to what occurs in the simple fracture model.

For the model where one sets $m$ to the value of 2.5 , which is the most realistic parameterization, Fig. 8 tells us, following the same argumentation as for the $m=4.5$ case, that the immobile domain heterogeneity weakly controls the mobile domain transport.

\subsubsection{On the control of the mobile domain transport on the trapping rate}

Fig. 9 shows that the trapping rate $\gamma$ is not constant but depends on the mobile time $t_{m}$. The function $\gamma(t)$ depends on the properties of the immobile domain controlled by $\kappa$ and $\zeta$ but also on the Pe value which means that this function $\gamma\left(t_{m}\right)$ is not an intrinsic property of the system, but depends on the flow rate. Yet we observed, for instance for the immobile domain characterized by $m=2.5$ and $\xi=0.1$ that the trapping rate is actually constant for value of $t_{m}$ larger than $200 \mathrm{~s}$ (materialized 


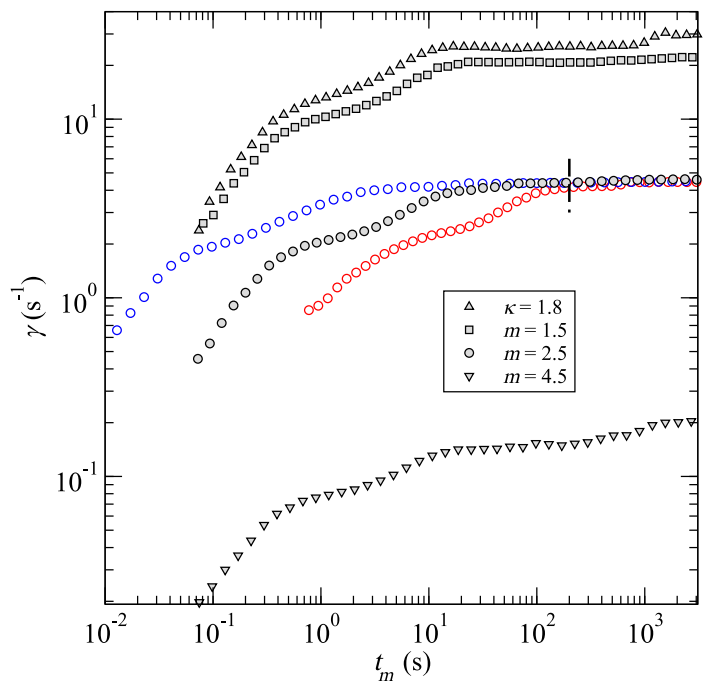

Fig. 9. $\gamma(t)$ versus $t_{m}$, for different tortuosity model for the immobile domain (with $\zeta=0.1$ ). Gray filled symbols are results for $P e=100$ while red and blue circles are results (for the $m=2.5$ model) for $P e=10$ and 1000, respectively. (For interpretation of the references to colour in this figure legend, the reader is referred to the web version of this article.)

by the vertical dashed line in Fig. 9). The system behaves as a constant trapping rate for range of $t_{m}$ which increases as $m$ increases.

Fig. 10 compares the theoretical conditional PDF $P_{n}\left(n \mid t_{m}\right)$ assuming a Poisson distribution following Eq. 22 where the trapping rate is a function of $t_{m}$ using the values given in Fig. 9 with the conditional PDF $P_{n}\left(n \mid t_{m}\right)$ resulting from the MC10 simulations. It can be seen that using the $\gamma\left(t_{m}\right)$ function reestablishes the consistency with the compound Poisson process model (compared to Fig. 6). This gives us the sound basis for implementing the CTRW approach presented in Section 3.2, but implemented with the $\gamma\left(t_{m}\right)$ function, for upscaling the transport in the MC10 sample.

\section{CTRW upscaling of MC10}

As shown above, the dependence of the trapping rate $\gamma$ on the time spent by a particle in the mobile domain $t_{m}$ is a critical feature triggered by the heterogeneity of the mobile-immobile domain interface. Accordingly, the proposed model is based on the implementation of the mobiletime dependence of the trapping rate in the CTRW upscaling model that was used to model the transport in the single fracture in Section 3.2. One speculates that the number of trapping events $n_{t_{m}}$ during a mobile transition of duration $t_{m}$ is Poisson-distributed in which the trapping rate is given by the $\gamma\left(t_{m}\right)$ function. To test this assumption we build a simple upscaled model in which the transport processes is modeled by the unconditional downstream motion of particles with constant distance $L_{z}$ and a random transition time $t_{m}$ distributed according to $\breve{\psi}_{t_{m}}$ (plotted in Fig. 8). The first passage time for each particle injected at $t=0$ is similar to Equation (9)

$t_{t}=t_{m}+\sum_{i=1}^{n_{t}} \tau_{i m}$

Here the number of trapping events $n_{t}$ is a random variable distributed according to the Poisson distribution

$P\left(n \mid t_{m}\right)=\frac{\left(\left\langle n_{t}\right\rangle\right)^{n} \exp \left(-\left\langle n_{t}\right\rangle\right)}{n !}$,

with $\left\langle n_{t}\right\rangle=t_{m} \gamma\left(t_{m}\right)$. The trapping time $\tau_{i m}$ is also a random variable distributed according to $\breve{\psi}_{\tau_{i m}}$. The results of the upscaled CTRW model are first compared with those obtained with the UPSCAL TDRW simulations in the left plot of Fig. 11. The UPSCAL TDRW simulations integrate the same relocation process as in the upscaled CTRW model. As such, and because both the models share the same mobile time distribution $\check{\psi}_{t_{m}}$ computed assuming no immobile domain, the comparison of the CTRW with the TDRW UPSCAL model is a sound validation of the approach used to model the trapping rate and the trapping time in the immobile domain, independently of retro-action of the immobile domain on the mobile domain time distribution. The results given in Fig. 11 show that the CTRW is perfectly reproducing the BTC computed with the TDRW UPSCAL model for different properties of the immobile domain. The comparison of the BTCs computed with the CTRW model using the mobile time distribution $\check{\psi}_{t_{m}}$ resulting from the FULL TDRW model with those computed with the TDRW are given in Fig. 11 (right plot) for different immobile domain parameters, as well as for different values of $P e$ in Fig. 12. As expected, the CTRW model does not reproduce well the TDRW data for the case where $m=1.5$ and $\zeta=0$ for which the immobile domain provides the largest opportunity for particle to shortcut the main flow streams. Clearly this specific process cannot be taken into account by the Lagrangian CTRW model. Conversely, we observe a good fit of the upscaled CTRW model results with those computed by the direct TDRW simulations for the reference case where $\kappa(\mathbf{x})=\phi(\mathbf{x})^{1-m}$ with $m=$ 2.5. Specifically, the upscaled model reproduces perfectly the data for long times $\left(t \geq 10^{2} \mathrm{~s}\right)$ for both $\zeta=0$ and 0.1 while the fit at intermediate times is better for the case where the porosity threshold is applied, i.e. $\zeta=0.1$. The ability of the CTRW model to reproduce the direct simulations for times ranging over six orders of magnitude and for different values of the Pe number is shown in Fig. 12. This figure also displays the PDFs $\psi_{t_{t}}$ computed by the CTRW model assuming a single trapping rate value $\gamma=\langle\gamma(p)\rangle$ thus allowing us to figure out the noticeable improvement of using the temporally evolving $\gamma\left(t_{m}\right)$ for upscaling the BTCs at long times.

\section{Conclusions}

With the objective of upscaling transport in heterogeneous media, a Lagrangian transport model that separates the processes of transport in mobile and immobile domains is presented along with its particle-based CTRW implementation. Assuming an homogeneous immobile domain and a continuous mobile-immobile interface, mass transfer occurs as a compound Poisson process with constant mobile-immobile exchanges rate. We show that this model is equivalent to the multirate mass transfer model of Haggerty and Gorelick (1995) with the mean trapping event number of the Lagrangian model being equal to the capacity ratio of the multirate mass transfer model. In other words, the transport process in the Lagrangian CTRW model is characterized by the mean trapping event number which also denotes the ratio of the solute mass in the immobile domain to that in the mobile domain at equilibrium. We show that this 1-dimensional CTRW model perfectly reproduces the direct 2dimensional TDRW simulations of the transport of solute in a linear fracture embedded into a porous matrix.

However, this simple upscaled model is not able to reproduce accurately the trans- port in the digitalized carbonate sample MC10 that is used to illustrate highly heterogeneous porous media for which we performed direct 3- dimensional TDRW simulations. Yet, the direct simulations allow the thorough statistical analysis of the mass transfer dynamics within the two domains and of the mass exchanged at their interface which is spatially discontinuous and heterogeneous in terms of trapping rate. We found that this heterogeneity of the mobile-immobile interface, together with the complexity of the flow in the mobile domain causes a deviation from the CTRW model presented in Section 3.2 or equivalently a deviation from the MRMT model.

For instance, the discrepancy between the computed survival time distribution for MC10 and the exponential distribution characterizing the single trapping rate model arises from the non-uniqueness of the trapping rate; $\gamma$ is a function of $t_{m}$ (Fig. 9). The survival time distribution displays a power law trend for short survival times which denotes the superposition of exponential distributions of $\tau_{s}\left(t_{m}\right)$ with distinct av- 

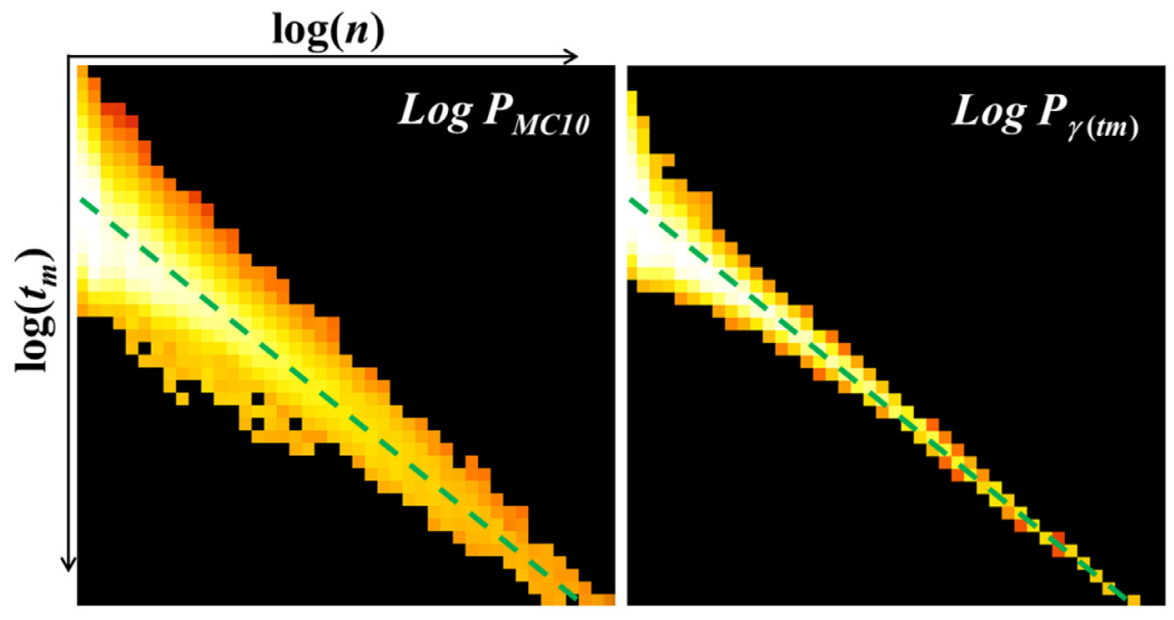

Fig. 10. Left: conditional probability $\log _{10}\left(P_{n}\left(n \mid t_{m}\right)\right)$ obtained for the MC10 sample. Right: conditional probability $\log _{10}\left(P_{n}\left(n \mid t_{m}\right)\right)$ corresponding to the theoretical Poisson distribution computed using the function $\gamma\left(t_{m}\right)$ displayed in Fig. 9. Black color indicates values of $\log _{10}\left(P_{n}\left(n \mid t_{m}\right)\right)$ smaller than -5 while color scale from dark red to white denotes values of $\log _{10}\left(P_{n}\left(n \mid t_{m}\right)\right)$ ranging from -5 to -0.68 . The green dashed line is a visual landmark identical to Fig. 6. Results are given for $P e=100, \kappa(\mathbf{x})=\phi(\mathbf{x})^{1-m}$ with $m=2.5$ and $\zeta=0.1$. (For interpretation of the references to colour in this figure legend, the reader is referred to the web version of this article.)
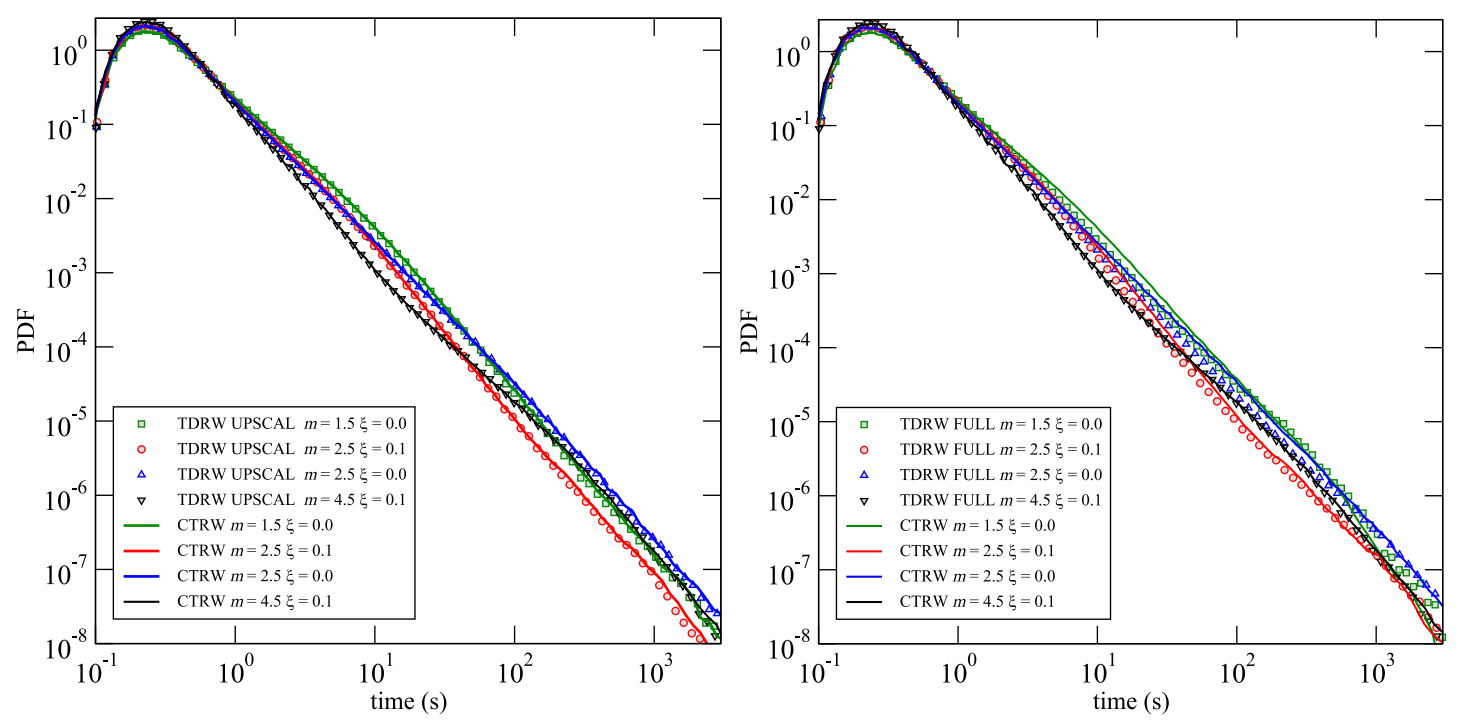

Fig. 11. The figure on the left displays the comparison of PDFs of the total $t_{t} \check{\psi}_{t}$, computed by the TDRW for the UPSCAL model with $\psi_{t}$, the total time PDFs resulting from the CTRW using as input the mobile time PDFs $\check{\psi}_{t_{m}}$ computed by the TDRW model without immobile domain (corresponding to the identical gray filled circle curve displayed in all plots of Fig. 8). The figure on the right displays the comparison of PDFs of the total $t_{t} \check{\psi}_{t}$ computed by the TDRW for the FULL model with $\psi_{t_{t}}$ the total time PDFs resulting from the CTRW using as input the mobile time PDFs $\check{\psi}_{t_{m}}$ computed by the FULL TDRW model (corresponding to the immobile-domain-properties-dependent black line curves displayed in Fig. 8).

erage: $\left\langle\tau_{s}\right\rangle\left(t_{m}\right) \equiv 1 / \gamma\left(t_{m}\right)$. Conversely, the increase of the occurrence of larger survival times compared to the exponential distribution denotes the lacunarity of the mobile-immobile interface; the distance (and thus the time) between two trapping events can be augmented due to the absence of available immobile domain in some parts of the mobile domain.

Introducing this functional dependence of the trapping rate to the mobile time allows complying with a mobile time dependent compound Poisson process. In other words, the mass transfers at the scale of the sample can be modeled as the ensemble average of residence-timedependent mass transfers that can individually be modeled as single rate processes.

The comparison of the upscaled model against the direct 3dimensional TDRW for different assumed properties of the immobile domain and different values of the Péclet number permits to prove the efficiency of the model to reproduce the complex mass transfers in the two domains and at their interface, as long as the spreading of solute due to the immobile domain does not reach a level where it produces a strong decorrelation of the velocity experienced by the particles in the mobile domain. This situation occurs when immobile domain clus- ters allow short-cut connections between zones of the mobile domain displaying distinctly different flow rates. Fortunately, this situation is quite unlikely in reservoir rocks since the diffusivity in the immobile domain is generally decreasing from the mobile-immobile interface ensuring together with the presence of non-diffusing zones a certain insulation between adjacent flowing pore networks.

The final conclusion of this study is that the proposed upscaled Lagrangian transport model provides an accurate description of the observed non-Fickian breakthrough curves in heterogeneous dual-porosity media, even when they are displayingbroad distributions of flow velocity values and highly heterogeneous immobile zones, such as the carbonate example studied here. This model is a dual multirate mass transfer model (DMRMT), in which the multiple rates of trapping arise from both the heterogeneity of the diffusion in immobile domain and the heterogeneity of the flow in the mobile domain.

\section{Acknowledgments}

PG and DR acknowledge funding from the CNRSIEA through the project CROSSCALE (ex-PICS $n \backslash 260280090$ ). MD and AP acknowledge 


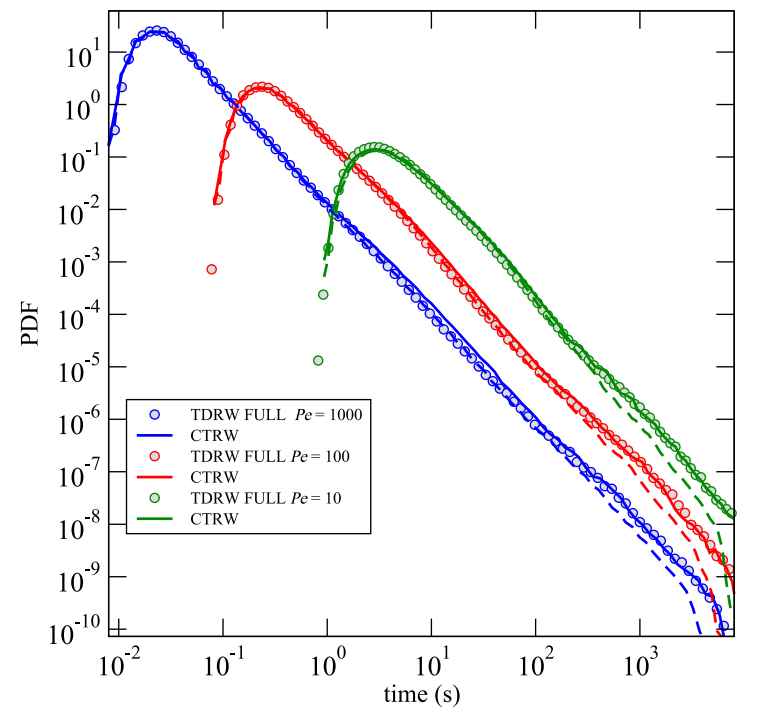

Fig. 12. Comparison of PDFs of the total $t_{t} \check{\psi}_{t_{t}}$ computed using the TDRW for the FULL model with those resulting from the CTRW using as input the mobile time PDFs $\check{\psi}_{t_{m}}$ computed using the TDRW model for $P e=10,100$ and 1000 for the case where $\kappa(\mathbf{x})=\phi(\mathbf{x})^{1-m}$ with $m=2.5$ and $\zeta=0.1$. The dashed lines display the results of the CTRW using constant trapping rate $\gamma=1.51$.

funding from the Spanish Ministry of Science and Innovation through the project HydroPore (PID2019-106887GB-C31).

\section{Declaration of Competing Interest}

The authors declare that they have no known competing financial interests or personal relationships that could have appeared to influence the work reported in this paper.

\section{Appendix A. MC10 sample description}

MC10 sample is a reefal carbonate sample from Llucmajor platform (Majorca, Spain) and is chosen as a typical example of complex porous media. Carbonate reservoirs are known to be heterogeneous over a wide range of scales as the result of the deposition and multiple diagenetic processes. The sample is a cylinder of $9 \mathrm{~mm}$ diameter that was imaged using X-ray computed microtomography which is a nondestructive imaging technique that produces 3-dimensional images from a set (usually more than 1500) 2D X-ray attenuation images taken at different angles. The sample was imaged at the ID19 beamline of the European Synchrotron Radiation Facility (ESRF), Grenoble, France. The 3dimensional reconstruction process that was performed using the single distance phase retrieval algorithm described by Paganin et al. (2002) applying an unsharp filter before reconstruction. The final 3-dimensional image is formed of cubic voxels associated with a value quanitifying the $\mathrm{X}$-ray attenuation integrated over the voxel volume. This value ranges from that corresponding to the X-ray attenuation of air for voxels belonging to macropores to that corresponding to the X-ray attenuation of the solid rock, i.e. where porosity is zero. The voxel size denotes the optical resolution of the image. The total X-ray energy attenuation depends on the attenuation of the fraction of the rock-forming minerals and of the porosity. For a single component solid phase such as the sample considered here that is made of calcite, the X-ray energy attenuation integrated over each voxel of the final 3-dimensional image denotes the porosity. The MC10 sample was previously investigated by Smal et al., 2018 for illustrating the application of a new segmentation algorithm allowing mapping the unresolved porosity, i.e. the fraction of the pore space containing pores that are smaller than the imaging resolution. While connected macropores allow solute transport by advection and diffusion, solute transport in the microporous material is assumed to be controlled by diffusion. Accordingly, connected macro-porosity delimits the mobile domain while the microporous material delimits the immobile domain. Applying the methodology proposed by Smal et al., 2018 one obtains a segmented image formed of a connected mobile domain, an immobile domain which in our case is almost completely surrounding the mobile domain and patches of solid material where no solute transport occurs (see Fig. 1). Note that the immobile domain contains isolated macropores (voxels of porosity equal to 1) corresponding to the non-connected macro-porosity. The 3-dimensional image used in this paper to illustrate mobile-immobile solute transport and its upscaling is a cubic sub-volume of side size $900 \times 900 \times 900$ cubic voxels. The voxel size is $1.6867 \times 10^{-6} \mathrm{~m}$. The average pore size is evaluated from the distribution of the chord-length distribution function (Torquato and $\mathrm{Lu}, 1993)$ as $70 \times 10^{-6} \mathrm{~m}$. Accordingly, the mobile domain is formed by around $10^{4}$ pores.

This $900 \times 900 \times 900$ image is obtained from a volume of $300 \times 300 \times 300$ voxels cropped from the segmented image of the cylindrical rock sample, then having each voxel divided by 3 in each of the directions ( 1 voxel is meshed in 27 voxels). This procedure allows obtaining a sufficient resolution for an accurate calculation of the Stokes flow.

\section{Appendix B. Immobile domain properties}

Different tortuosity models are investigated assuming a porosity threshold of $\zeta=0.1$ and tortuosity defined by $\kappa(\phi)=\phi^{1-m}$ with $m=1.5$, 2.5 and 4.5 as well as with a constant tortuosity model $\kappa=1.8$ and $\zeta=0$. Table B.1 displays the average effective diffusion $\left\langle D_{e}\right\rangle$ and a geometric evaluation of the diffusion characteristic time $t_{d}=\ell_{i m}^{2} /\left(2\left\langle D_{e}\right\rangle\right)$ for these different models, with $\ell_{i m}$ approximated by the ratio of the immobile volume to the mobile-immobile interface area.

The immobile domain transport properties (by diffusion) can also be characterized by the memory function $\varphi(t)$ that denotes the probability that a particle entering the immobile zone at $t=0$ remains there until time $t$. For a given digitized rock sample segmented into mobile and immobile domains, $\varphi(t)$ characterizes the geometry and the volume fraction of the immobile domain and the topology of the mobile-immobile interface (Gouze et al., 2008). $\varphi(t)$ is derived from the trapping time $\operatorname{PDF} \check{\psi}_{\tau_{i m}}$ with the relation $\varphi(t)=1-\int_{0}^{t} \check{\psi}_{\tau_{i m}}\left(t^{\prime}\right) \mathrm{d} t^{\prime}$.

As shown in Fig. B.13, the assumptions made on the formulation of the tortuosity and its parameterization (Equations (1) - (3)) are noticeably modifying $\varphi(t)$. Specifically, one can see that increasing the exponent $m$ of the tortuosity model (Equation (2)) and implementing a porosity threshold $\zeta>0$ changes the shape and the average slope of the memory function, and therefore should have a critical effect on the overall hydrodynamic transport in the sample. The characteristic diffusion time in the immobile domain $t_{c}$ is given by the mean trapping time $\left\langle\check{\psi}_{\tau_{i m}}\right\rangle$. Table B.1 displays the values of $t_{c}$ for these different models. The model with constant tortuosity $(\kappa(\mathbf{x})=1.8)$ and the model with $\kappa(\mathbf{x})=\phi(\mathbf{x})^{1-m}$ with $m=1.5$ display similar values of $t_{c}$. Conversely, increasing the exponent $m$ increases noticeably the characteristic diffusion time, i.e. lengthen the effective diffusion path length, whereas applying a threshold $(\zeta=0.1)$ reduces the immobile domain extension and consequently the value of $t_{c}$. The ratio $t_{d} / t_{c}$ is given in Table B.1. This ratio is a qualitative indication of the effective intricacy and heterogeneity of the diffusion paths characterized by the mean diffusion time $t_{c}$ compared to the mean diffusion time expected from a simple geometry of the immobile domain of similar volume and mobile-immobile interface area. When all the porosity of the immobile domain is considered $(\zeta=$ 0.0 ) the ratio $t_{d} / t_{c}$ is ranging from 22 to 37 emphasizing the strong complexity of the diffusion path (that can be guessed from the cross section presented in Fig. 1). When the zones of the smallest porosity values are not participating to diffusion $(\zeta=0.1)$ the ratio $t_{d} / t_{c}$ is much smaller $\left(t_{d} / t_{c}=5.8 \pm 0.5\right)$ and apparently independent of the tortuosity model, 


\section{Table B.1}

Mean properties of the immobile domain models characterized by the porosity threshold $\zeta$ and the tortuosity either constant $(\kappa(\mathbf{x})=1.8)$ or function of porosity $\left(\kappa(\mathbf{x})=\phi(\mathbf{x})^{1-m}\right.$ with $m=1.5,2.5$ and 4.5$)$. Colored boxes denotes the parameter sets used for the results discussed in Section 4.

\begin{tabular}{|c||c|c|c|c|c|c|c|c|}
\hline \multicolumn{1}{|c||}{} & \multicolumn{2}{c|}{$\kappa=1.8$} & \multicolumn{2}{c|}{$m=1.5$} & \multicolumn{2}{c|}{$m=2.5$} & \multicolumn{2}{c|}{$m=4.5$} \\
\hline$\zeta(-)$ & 0.0 & 0.1 & 0.0 & 0.1 & 0.0 & 0.1 & 0.0 & 0.1 \\
\hline \hline$\left\langle D_{e}\right\rangle\left(10^{-12} \mathrm{~m}^{2} / \mathrm{s}\right)$ & 59.9 & 98.1 & 39.7 & 74.0 & 6.2 & 13.5 & 0.2 & 0.5 \\
\hline$t_{d}(\mathrm{~s})$ & 3.3 & 0.3 & 5.0 & 0.5 & 31.9 & 2.5 & 988.3 & 395.3 \\
\hline$t_{c}=\left\langle\psi_{\tau_{i m}}^{\circ}\right\rangle(\mathrm{s})$ & 0.15 & 0.061 & 0.18 & 0.075 & 0.96 & 0.39 & 26.7 & 12.8 \\
\hline$t_{d} / t_{c}(-)$ & 22.0 & 5.70 & 27.1 & 6.2 & 33.2 & 6.4 & 37.0 & 5.3 \\
\hline
\end{tabular}

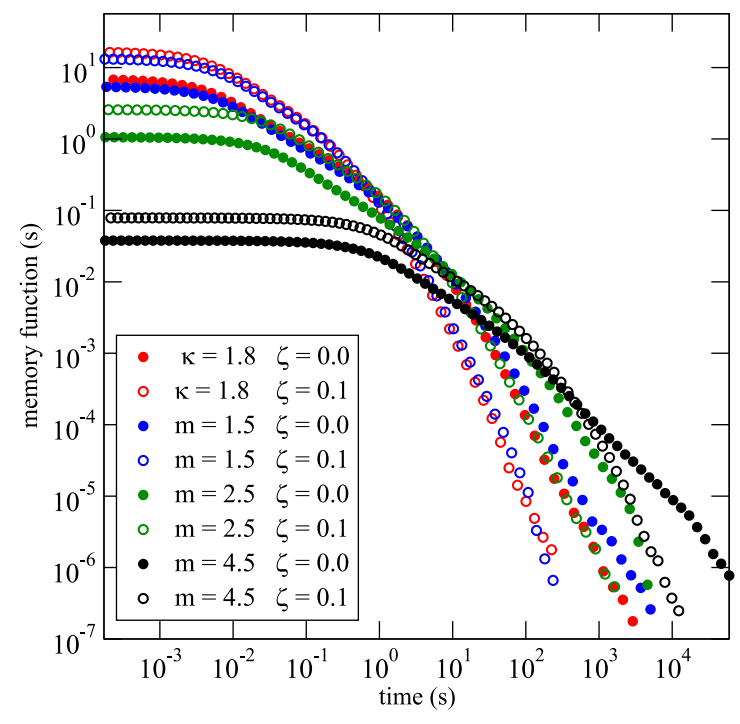

Fig. B.13. The memory function $\varphi(t)$ for different properties of the immobile domain: for constant tortuosity $(\kappa=1.8)$ and for $\kappa(\phi)=\phi^{1-m}$ with $m$ ranging from 1.5 to 4.5 , without and with porosity threshold, i.e. $\zeta=0$ and $\zeta=0.1$, respectively.

suggesting that applying the porosity threshold acts as decreasing the effective intricacy of the diffusion paths.

Appendix C. Vertical averaging of the mobile-immobile model of a single fracture embedded in an homogeneous matrix

Flux continuity at the fracture-matrix interface implies

$\left.\phi_{m} D_{m} \frac{\partial c_{m}(\mathbf{x}, t)}{\partial y}\right|_{y=0}=\left.\phi_{i m} D_{i m} \frac{\partial c_{i m}(y, t)}{\partial y}\right|_{y=0}$,

such that

$\phi_{m} \frac{\partial \bar{c}_{m}(x, t)}{\partial t}+q \frac{\partial \bar{c}_{m}(x, t)}{\partial x}-\phi_{m} D_{m} \frac{\partial^{2} \bar{c}_{m}(x, t)}{\partial x^{2}}=-\left.\frac{1}{d_{m}} \phi_{i m} D_{i m} \frac{\partial c_{i m}(y, t)}{\partial y}\right|_{y=0}$

The flux over the interface is obtained by integration of (13) over $y$, which gives

$\left.\phi_{m} D_{m} \frac{\partial c_{m}(y, t)}{\partial y}\right|_{y=0}=\phi_{i m} d_{i m} \frac{\partial \bar{c}_{i m}(x, t)}{\partial t}$.

The matrix concentration is obtained from (13). In Laplace space, we obtain

$c_{i m}^{*}(y, \lambda)=\frac{\cosh \left[\left(y+d_{i m}\right) \sqrt{\lambda / D_{i m}}\right]}{\cosh \left(\sqrt{\lambda \tau_{D}}\right)} \bar{c}_{m}^{*}(x, \lambda)$, where we defined $\tau_{D}=d_{i m}^{2} / D_{i m}$. The average matrix concentration is then obtained by integration over $y$, which gives

$\bar{c}_{i m}^{*}(y, \lambda)=\varphi^{*}(\lambda) \bar{c}_{m}^{*}(x, \lambda)$,

where the memory function is defined by (16). The memory function at $\lambda=0$ is $\varphi^{*}(0)=1$, which means

$\int_{0}^{\infty} d t \varphi(t)=1$.

\section{Appendix D. Upscaled Lagrangian model}

The particle density in the Lagrangian model is obtained from CTRW theory (Berkowitz et al., 2006; Russian et al., 2016) as

$c_{i}(t)=\int_{0}^{t} d t^{\prime} R_{i}\left(t^{\prime}\right) \int_{0}^{t-t^{\prime}} d t^{\prime \prime} \psi\left(t^{\prime \prime}\right), R_{i}(t)=\delta_{i, 0}+\int_{0}^{t} d t^{\prime} \psi\left(t-t^{\prime}\right)\left[w_{u} R_{i+1}\left(t^{\prime}\right)+w_{d} R_{i-1}\right.$

These equations are combined into a single generalized master equation in Laplace space

$\lambda c_{i}^{*}(\lambda)=\delta_{i, 0}+\frac{\lambda \psi^{*}(\lambda)}{1-\psi^{*}(\lambda)}\left[w_{u} c_{i-1}^{*}(\lambda)+w_{d} c_{i+1}^{*}(\lambda)-c_{i}^{*}(\lambda)\right]$.

Inserting (23) into (D.3) and using the definition (18) of the transition probabilities, we obtain

$\lambda c_{i}^{*}(\lambda)=\delta_{i, 0}+\frac{D_{m} \frac{c_{i-1}^{*}(\lambda)+c_{i+1}^{*}(\lambda)-2 c_{i+1}^{*}(\lambda)}{\ell^{2}}-v \frac{c_{i+1}^{*}(\lambda)-c_{i}^{*}(\lambda)}{\ell}}{1+\lambda^{-1} \gamma\left[1-\psi_{i m}^{*}(\lambda)\right]}$.

In the limit $\ell \rightarrow 0$, we obtain

$\lambda c^{*}(x, \lambda)=\delta(x)+\frac{D_{m} \frac{\partial^{2} c^{*}(x, \lambda)}{\partial x^{2}}-v \frac{c^{*}(x, \lambda)}{\partial x}}{1+\lambda^{-1} \gamma\left[1-\psi_{i m}^{*}(\lambda)\right]}$.

The concentration $c^{*}(x, \lambda)$ denotes the sum of the fracture and matrix concentrations $c^{*}(x, \lambda)=c_{m}^{*}(x, \lambda)+c_{i m}^{*}(x, \lambda)$. We identify

$c_{i m}^{*}(x, \lambda)=\gamma \vartheta^{*}(\lambda) c_{m}^{*}(x, \lambda)$

where the memory kernel $\vartheta(t)$ is defined through its Laplace transform as

$\vartheta^{*}(\lambda)=\frac{1}{\lambda}\left[1-\psi_{i m}^{*}(\lambda)\right]$.

Using (D.6) and (D.7) in (10), we obtain for $c_{m}^{*}(x, \lambda)$

$\lambda c_{m}^{*}(x, \lambda)+v \frac{\partial c_{m}^{*}(x, \lambda)}{\partial x}-D_{m} \frac{\partial^{2} c_{m}^{*}(x, \lambda)}{\partial x^{2}}=\delta(x)-\lambda c_{i m}^{*}(x, \lambda)$.

Finally, inverse Laplace transform of (D.8) gives (24).

\section{Supplementary material}

Supplementary material associated with this article can be found, in the online version. 


\section{CRediT authorship contribution statement}

Philippe Gouze: Conceptualization, Software, Formal analysis, Investigation, Methodology, Writing - original draft, Writing - review \& editing. Alexandre Puyguiraud: Formal analysis, Methodology, Writing - review \& editing. Delphine Roubinet: Formal analysis, Methodology, Writing - review \& editing. Marco Dentz: Conceptualization, Formal analysis, Methodology, Writing - original draft, Writing - review \& editing.

\section{References}

Ahmadi, A., Quintard, M., Whitaker, S., 1998. Transport in chemically and mechanically heterogeneous porous media. Adv Water Resour 22 (1), 59-86. https://doi.org/10.1016/s0309-1708(97)00032-8.

Alim, K., Parsa, S., Weitz, D.A., Brenner, M.P., 2017. Local pore size correlations determine flow distributions in porous media. Phys. Rev. Lett. 119 (14). https://doi.org/10.1103/physrevlett.119.144501.

Aramideh, S., Vlachos, P.P., Ardekani, A.M., 2018. Pore-scale statistics of flow and transport through porous media. Physical Review E 98 (1). https://doi.org/10.1103/physreve.98.013104.

Archie, G., 1942. The electrical resistivity log as an aid in determining some reservoir characteristics. Transactions of The American Institute of Mining and Metallurgical Engineers 146, 54-61.

Bear, J., 1972. Dynamics of fluids in porous media. American Elsevier, New York.

Benson, D.A., Meerschaert, M.M., 2009. A simple and efficient random walk solution of multi-rate mobile/immobile mass transport equations. Adv. Wat. Res. 32 (4), $532-539$.

Berkowitz, B., Cortis, A., Dentz, M., Scher, H., 2006. Modeling non-fickian transport in geological formations as a continuous time random walk. Rev. Geophys. 44 (2).

Bijeljic, B., Blunt, M.J., 2006. Pore-scale modeling and continuous time random walk analysis of dispersion in porous media. Water Resour Res 42 (1).

Bijeljic, B., Mostaghimi, P., Blunt, M.J., 2011. Signature of non-fickian solute transport in complex heterogeneous porous media. Phys. Rev. Lett. 107 (20), 204502.

Bijeljic, B., Mostaghimi, P., Blunt, M.J., 2013. Insights into non-fickian solute transport in carbonates. Water Resour Res 49 (5), 2714-2728. https://doi.org/10.1002/wrcr.20238.

Brenner, H., 1980. Dispersion resulting from flow through spatially periodic porous media. Proc. Roy. Soc. A 297, 81-133.

Carrera, J., Sanchez-Vila, X., Benet, I., Medina, A., Galarza, G., Guimera, J., 1998. On matrix diffusion: formulations, solution methods and qualitative effects. Hydrogeol J 6 (1), 178-190. https://doi.org/10.1007/s100400050143.

Ceriotti, G., Russian, A., Bolster, D., Porta, G., 2019. A double-continuum transport model for segregated porous media: derivation and sensitivity analysis-driven calibration. Adv Water Resour 128, 206-217. https://doi.org/10.1016/j.advwatres.2019.04.003.

Cherblanc, F., Ahmadi, A., Quintard, M., 2007. Two-domain description of solute transport in heterogeneous porous media: comparison between theoretical predictions and numerical experiments. Adv Water Resour 30 (5), 1127-1143.

Comolli, A., Hidalgo, J.J., Moussey, C., Dentz, M., 2016. Non-fickian transport under heterogeneous advection and mobile-immobile mass transfer. Transp Porous Media 115 (2), 265-289.

Cortis, A., Berkowitz, B., 2004. Anomalous transport in "classical" soil and sand columns. Soil Sci. Soc. Am. J. 68 (5), 1539. https://doi.org/10.2136/sssaj2004.1539.

Davit, Y., Bell, C.G., Byrne, H.M., Chapman, L.A., Kimpton, L.S., Lang, G.E., Leonard, K.H., Oliver, J.M., Pearson, N.C., Shipley, R.J., Waters, S.L., Whiteley, J.P., Wood, B.D., Quintard, M., 2013. Homogenization via formal multiscale asymptotics and volume averaging: how do the two techniques compare? Adv Water Resour 62, 178-206. https://doi.org/10.1016/j. advwatres.2013.09.006.

Davit, Y., Wood, B.D., Debenest, G., Quintard, M., 2012. Correspondence between one- and two-equation models for solute transport in two-region heterogeneous porous media. Transp Porous Media 95 (1), 213-238. https://doi.org/10.1007/s11242-012-0040-y.

De Anna, P., Le Borgne, T., Dentz, M., Tartakovsky, A.M., Bolster, D., Davy, P., 2013. Flow intermittency, dispersion, and correlated continuous time random walks in porous media. Phys. Rev. Lett. 110 (18), 184502.

De Anna, P., Quaife, B., Biros, G., Juanes, R., 2017. Prediction of velocity distribution from pore structure in simple porous media. Phys. Rev. Fluids 2, 124103. https://doi.org/10.1103/PhysRevFluids.2.124103.

Dentz, M., Gouze, P., Russian, A., Dweik, J., Delay, F., 2012. Diffusion and trapping in heterogeneous media: an inhomogeneous continuous time random walk approach. Adv Water Resour 49. https://doi.org/10.1016/j.advwatres.2012.07.015.

Dentz, M., Icardi, M., Hidalgo, J.J., 2018. Mechanisms of dispersion in a porous medium. J Fluid Mech 841, 851-882. https://doi.org/10.1017/jfm.2018.120.

Dentz, M., Kang, P.K., Comolli, A., Le Borgne, T., Lester, D.R., 2016. Continuous time random walks for the evolution of lagrangian velocities. Phys. Rev. Fluids 1 (7), 074004.

Domenico, P.A., Schwartz, F.W. (Eds.), 1997. Physical and chemical hydrogeology. Wiley.

Feller, W., 1968. An introduction to probability theory and its applications. Wiley Series in Probability and Statistics, 1 . Wiley.

Garing, C., Luquot, L., Pezard, P.A., Gouze, P., 2014. Electrical and flow properties of highly heterogeneous carbonate rocks. Am Assoc Pet Geol Bull 98 (1), 49-66. https://doi.org/10.1306/05221312134.

van Genuchten, M.T., Wierenga, P.J., 1976. Mass transfer studies in sorbing porous media $i$. analytical solutions. Soil Sci. Soc. Am. J. 40 (4), 473-480. https://doi.org/10.2136/sssaj1976.03615995004000040011x.
Gjetvaj, F., Russian, A., Gouze, P., Dentz, M., 2015. Dual control of flow field heterogeneity and immobile porosity on non-fickian transport in berea sandstone. Water Resour Res 51 (10), 8273-8293. https://doi.org/10.1002/2015WR017645.

Gouze, P., Melean, Y., Le Borgne, T., Dentz, M., Carrera, J., 2008. Non-fickian dispersion in porous media explained by heterogeneous microscale matrix diffusion. Water Resour Res 44 (11). https://doi.org/10.1029/2007WR006690.

Haggerty, R., Gorelick, S.M., 1995. Multiple-rate mass transfer for modeling diffusion and surface reactions in media with pore-scale heterogeneity. Water Resour Res 31 (10), 2383-2400. https://doi.org/10.1029/95WR10583.

Hebert, V., Garing, C., Luquot, L., Pezard, P.A., Gouze, P., 2015. Multi-scale x-ray tomography analysis of carbonate porosity. Geological Society, London, Special Publications 406 (1), 61-79. https://doi.org/10.1144/SP406.12.

Holzner, M., Morales, V.L., Willmann, M., Dentz, M., 2015. Intermittent lagrangian velocities and accelerations in three-dimensional porous medium flow. Phys. Rev. E 92, 013015.

Hommel, J., Coltman, E., Class, H., 2018. Porositypermeability relations for evolving pore space: a review with a focus on (bio-)geochemically altered porous media. Transp Porous Media 124, 589629. https://doi.org/10.1007/s11242-018-1086-2.

Hunt, A.G., Sahimi, M., 2017. Flow, transport, and reaction in porous media: percolation scaling, critical-path analysis, and effective medium approximation. Rev. Geophys. 55 (4), 993-1078. https://doi.org/10.1002/2017RG000558.

de Josselin de Jong, G., 1958. Longitudinal and transverse diffusion in granular deposits. Trans. Amer. Geophys. Un. 39, 67-74.

Jourak, A., Frishfelds, V., Hellström, J.G.I., Lundström, T.S., Herrmann, I., Hedström, A. 2013. Longitudinal dispersion coefficient: effects of particle-size distribution. Transp Porous Media 99 (1), 1-16. https://doi.org/10.1007/s11242-013-0159-5.

Kang, P.K., de Anna, P., Nunes, J.P., Bijeljic, B., Blunt, M.J., Juanes, R., 2014. Pore-scale intermittent velocity structure underpinning anomalous transport through 3-d porous media. Geophys Res Lett 41 (17), 6184-6190. https://doi.org/10.1002/2014GL061475.

Liu, Y., Kitanidis, P.K., 2012. Applicability of the dual-Domain model to nonaggregated porous media. Ground Water 50 (6), 927-934.

Margolin, G., Dentz, M., Berkowitz, B., 2003. Continuous time random walk and multirate mass transfer modeling of sorption. Chem Phys 295 (1), 71-80. https://doi.org/10.1016/j.chemphys.2003.08.007.

Matyka, M., Golembiewski, J., Koza, Z., 2016. Power-exponential velocity distributions in disordered porous media. Phys. Rev. E 93, 013110.

Morales, V.L., Dentz, M., Willmann, M., Holzner, M., 2017. Stochastic dynamics of intermittent pore-scale particle motion in three-dimensional porous media: experiments and theory. Geophys Res Lett 44 (18), 9361-9371.

Niemi, A., Bear, J., Bensabat, J. (Eds.), 2017. Geological Storage of CO2 in Deep Saline Formations. Springer Netherlands.

Nœetinger, B., Estebenet, T., 2000. Up-scaling of double porosity fractured media using continuous-time random walks methods. Transp Porous Media 39 (3), 315-337. https://doi.org/10.1023/A:1006639025910.

Orgogozo, L., Golfier, F., Bues, M.A., Quintard, M., Kone, T., 2013. A dual-porosity theory for solute transport in biofilm-coated porous media. Adv Water Resour 62 (3). https://doi.org/10.1016/j.advwatres.2013.09.011.

Paganin, D., Mayo, S., Gureyev, T.E., Miller, P.R., Wilkins, S.W., 2002. Simultaneous phase and amplitude extraction from a single defocused image of a homogeneous object. J Microsc 206 (1), 33-40.

Pfannkuch, H.O., 1963. Contribution a l'étude des déplacements de fluides miscibles dans un milieux poreux. Rev. Inst. Fr. Petr. 18, 215-270.

Poinssot, C., Geckeis, H. (Eds.), 2012. Radionuclide Behaviour in the Natural Environment. Woodhead Publishing.

Porta, G., Chaynikov, S., Riva, M., Guadagnini, A., 2013. Upscaling solute transport in porous media from the pore scale to dual- and multicontinuum formulations. Water Resour Res 49 (4), 2025-2039. https://doi.org/10.1002/wrcr.20183.

Porta, G.M., Bijeljic, B., Blunt, M.J., Guadagnini, A., 2015. Continuum-scale characterization of solute transport based on pore-scale velocity distributions. Geophys Res Lett 42 (18), 7537-7545. https://doi.org/10.1002/2015gl065423.

Puyguiraud, A., Gouze, P., Dentz, M., 2019a. Stochastic dynamics of lagrangian porescale velocities in three-dimensional porous media. Water Resour Res 55, 1196-1217. https://doi.org/10.1029/2018WR023702.

Puyguiraud, A., Gouze, P., Dentz, M., 2019a. Upscaling of anomalous pore-scale dispersion. Transp Porous Media 128, 837-855. https://doi.org/10.1007/s11242-019-01273-3.

Puyguiraud, A., Gouze, P., Dentz, M., 2020. Is there a representative elementary volume for anomalous dispersion? Transp Porous Media 131, 767-778. https://doi.org/10.1007/s11242-019-01366-z.

Quintard, M., Whitaker, S., 1994. Convection, dispersion and interfacial transport of contaminants: homogeneous media. Adv. Water Resour. 17, 221-239.

Rashidi, M., Peurrung, L., Tompson, A., Kulp, T., 1996. Experimental analysis of porescale flow and transport in porous media. Adv Water Resour 19 (3), 163-180. https://doi.org/10.1016/0309-1708(95)00048-8.

Russian, A., Dentz, M., Gouze, P., 2016. Time domain random walks for hydrodynamic transport in heterogeneous media. Water Resour Res 52 (5), 3309-3323. https://doi.org/10.1002/2015WR018511.

Saffman, P., 1959. A theory of dispersion in a porous medium. J Fluid Mech 6 (03), 321-349.

Salles, J., Thovert, J.-F., Delannay, R., Prevors, L., Auriault, J.-L., Adler, P.M., 1993. Taylor dispersion in porous media. determination of the dispersion tensor. Physics of Fluids A: Fluid Dynamics 5 (10), 2348-2376. https://doi.org/10.1063/1.858751.

Scheven, U., 2013. Pore-scale mixing and transverse dispersivity of randomly packed monodisperse spheres. Phys. Rev. Lett. 110 (21), 214504. 
Seymour, J.D., Gage, J.P., Codd, S.L., Gerlach, R., 2004. Anomalous fluid transport in porous media induced by biofilm growth. Phys. Rev. Lett. 93, 198103.

Siena, M., Riva, M., Hyman, J., Winter, C.L., Guadagnini, A., 2014. Relationship between pore size and velocity probability distributions in stochastically generated porous media. Physical Review E 89 (1), 013018.

Smal, P., Gouze, P., Rodriguez, O., 2018. An automatic segmentation algorithm for retrieving sub-resolution porosity from x-ray tomography images. Journal of Petroleum Science and Engineering 166, 198-207. https://doi.org/10.1016/j.petrol.2018.02.062.
Swanson, R.D., Binley, A., Keating, K., France, S., Osterman, G., Day-Lewis, F.D., Singha, K., 2015. Anomalous solute transport in saturated porous media: relating transport model parameters to electrical and nuclear magnetic resonance properties. Water Resour Res 51 (2), 1264-1283. https://doi.org/10.1002/2014wr015284.

Torquato, S., Lu, B., 1993. Chord-length distribution function for two-phase random media. Phys. Rev. E 47, 2950-2953. https://doi.org/10.1103/PhysRevE.47.2950.

de Vries, E.T., Raoof, A., van Genuchten, M.T., 2017. Multiscale modelling of dual-porosity porous media a computational pore-scale study for flow and solute transport. Adv Water Resour 105, 82-95. https://doi.org/10.1016/j.advwatres.2017.04.013. 